\title{
BASIC DOLBEAULT COHOMOLOGY AND WEITZENBÖCK FORMULAS ON TRANSVERSELY KÄHLER FOLIATIONS
}

\author{
SEOUNG DAL JUNG AND KEN RICHARDSON
}

\begin{abstract}
We study basic Dolbeault cohomology and find new Weitzenböck formulas on a transversely Kähler foliation. We investigate conditions on mean curvature and Ricci curvature that impose restrictions on basic Dolbeault cohomology. For example, we prove that on a transversely Kähler foliation with positive transversal Ricci curvature, there are no nonzero basic-harmonic forms of type $(r, 0)$, among other results.
\end{abstract}

\section{INTRODUCTION}

Let $\mathcal{F}$ be a foliation of a closed, smooth manifold $M$. One set of smooth invariants of $\mathcal{F}$ is the basic cohomology. The basic forms of $(M, \mathcal{F})$ are locally forms on the leaf space; that is, forms $\phi$ satisfying $X\lrcorner \phi=X\lrcorner d \phi=0$ for any vector $X$ tangent to the leaves, where $X\lrcorner$ denotes the interior product with $X$. Basic forms are preserved by the exterior derivative and are used to define basic de Rham cohomology groups $H_{B}^{*}$. In general these groups can be infinite-dimensional. A Riemannian foliation is a foliation on a smooth manifold such that the normal bundle $Q=T M / T \mathcal{F}$ may be endowed with a metric whose Lie derivative is zero along leaf directions. We can always extend this metric to a metric $g_{M}$ on $M$ that is called bundle-like, meaning that the leaves of $\mathcal{F}$ are locally equidistant. From this additional structure on the foliation, the basic Laplacian $\Delta_{B}$ is a version of the Laplace operator that preserves the basic forms. Many researchers have studied basic forms and the basic Laplacian on Riemannian foliations. It is well-known ([2], [11, [24, [31]) that on a closed oriented manifold $M$ with a transversally oriented Riemannian foliation $\mathcal{F}, H_{B}^{r} \cong \mathcal{H}_{B}^{r}$, where $\mathcal{H}_{B}^{r}=\operatorname{ker} \Delta_{B}$ is finite dimensional. Because of this Hodge theorem, researchers have been able to show relationships between curvature bounds and basic cohomology. In [15] J. Hebda proved that a lower bound on transversal Ricci curvature for a Riemannian foliation of a compact manifold causes the space of leaves to be compact and the first basic cohomology group to be trivial. Another example relating the geometry and the topology came in 1991, when M. Min-Oo et al. [26] proved that if the transversal curvature operator of $(M, \mathcal{F})$ is positive-definite, then the cohomology $H_{B}^{r}=\{0\}(0<r<q)$; that is, any basic-harmonic $r$-form is trivial. There are many other examples of known relationships between transversal curvature and basic cohomology.

We are interested in foliations that admit a transverse Kähler structure. These structures appear naturally in many situations. For example, Vaisman manifolds, Calabi-Eckmann

2010 Mathematics Subject Classification. 53C12; 53C21; 53C55; 57R30; 58J50.

Key words and phrases. Riemannian foliation, transverse Kähler foliation, Serre duality, Weitzenböck formula.

This paper was supported by the National Research Foundation of Korea (NRF) grant funded by the Korea government (MSIP) (NRF-2015R1A2A2A01003491). This work was also supported by a grant from the Simons Foundation (Grant Number 245818 to Ken Richardson). 
manfolds and Sasaki manifolds are not Kähler but carry a transverse Kähler structure. In particular, the characteristic foliation on a Sasaki manifold is transversely Kähler [5, Section 2] and is automatically homologically orientable [4, Proposition 7.2.3], causing many facts about Kähler manifolds to carry over. Being homologically orientable implies that the foliation is taut; there exists a metric such that the leaves of the foliation are minimal submanifolds. See [35] for a treatment. For instance, in [6], the authors were able to prove a hard Lefschetz theorem for compact Sasaki manifolds. In [8], the authors proved a similar result for cosymplectic manifolds. A major step in understanding the homologically orientable (taut) case was by A. El Kacimi-Alaoui in [10, Section 3.4], where the standard facts about Kähler manifolds and their cohomology are shown to carry over to the transverse structures and basic cohomology. Also, L. A. Cordero and R. A. Wolak [9] studied the properties of the basic cohomology on a taut transversely Kähler foliation by using the differential operator $\Delta_{T}$, which is different from $\Delta_{B}$. In fact, $\mathcal{F}$ is minimal (taut) if and only if $\Delta_{T}=\Delta_{B}$. Many known facts about Kähler forms $\omega$ carry over to the transversal geometry setting, but not all; for example, if the foliation is not taut, the powers $\omega^{k}$ need not generate nonzero basic cohomology classes. In the general case, in [19] S. D. Jung and J. S. Pak proved a Weitzenböck formula for the Dolbeault Laplacian and deduced among other results that when the mean curvature of the foliation is basic-harmonic, nonnegativity of the transverse Ricci curvature (and positivity at one point) implies that the first basic cohomology group is trivial. In [20], they showed that under some positivity assumptions on transverse curvature on a complete transversely Kähler foliation, the second basic cohomology group is generated by the Kähler form. In [18], S. D. Jung and H. Liu proved that if the transversal Ricci curvature of a compact transversely Kähler foliation is nonpositive and negative at one point, then there are no nonzero basic transverse holomorphic vector fields. G. Habib and L. Vezzoni showed a similar result by different methods in [14, Theorem 3.3]. They used a twisted version of the basic Dolbeault Laplacian and calculated the corresponding Weitzenböck formula. In another recent paper [25], Y. Kordyukov, M. Lejmi, and P. Weber showed that for a manifold with bundle-like, transversely Kähler metric for a taut foliation, under some conditions on the basic cohomology of one-forms, the basic version of the Seiberg-Witten invariants must be nontrivial.

In this paper, we study basic Dolbeault cohomology and give a new Weitzenböck formula for the basic Dolbeault Laplacian in foliations with a transverse Kähler structure, and in particular we are interested in general results that apply whether or not the foliation is taut. This paper is organized as follows: In Section 2, we recall the basic properties of Riemannian foliations, basic cohomology, and the basic Laplacian. In particular, we review the de Rham decomposition, Poincaré duality, the generalized Weitzenböck formula and some vanishing theorems. In Section 3, we review the many differential operators on transversely Kähler foliations and recall some known results, providing short proofs in some cases for the reader's benefit. If $\mathcal{F}$ is a minimal transversely Kähler foliation, then a basic holomorphic form of type $(r, 0)$ is basic-harmonic (Corollary 3.20). We prove a generalized Dolbeault decomposition (Theorem 3.21). Also, on a transversely Kähler foliation, the Serre duality theorem does not necessarily hold unless $\mathcal{F}$ is minimal, but we exhibit a version of Kodaira-Serre duality that actually does hold in all cases (Theorem 3.22, Corollary 3.23, and Example 3.26). In Section 4, we establish some new Weitzenböck formulas (Proposition 4.2) for the basic Dolbeault Laplacians and prove some vanishing results (Theorem 4.9 and Corollary 4.10). 


\section{Preliminaries}

Let $\left(M, g_{Q}, \mathcal{F}\right)$ be a $(p+q)$-dimensional Riemannian foliation of codimension $q$. Here, $g_{Q}$ is a holonomy invariant metric on the normal bundle $Q=T M / T \mathcal{F}$, meaning that $\mathcal{L}_{X} g_{Q}=0$ for all $X \in T \mathcal{F}$, where $\mathcal{L}_{X}$ denotes the Lie derivative with respect to $X$. Next, let $g_{M}$ be a bundle-like metric on $M$ adapted to $g_{Q}$. This means that if $T \mathcal{F}^{\perp}$ is the $g_{M}$-orthogonal complement to $T \mathcal{F}$ in $T M$ and $\sigma: Q \rightarrow T \mathcal{F}^{\perp}$ is the canonical bundle isomorphism, then $g_{Q}=\sigma^{*}\left(\left.g_{M}\right|_{T \mathcal{F} \perp}\right)$.

Let $\pi: T M \rightarrow Q$ be the bundle projection. The transversal Levi-Civita connection $\nabla$ on $Q \rightarrow M$ is given for all $s \in \Gamma(Q)$ by

$$
\nabla_{X} s= \begin{cases}\pi\left(\left[X, Y_{s}\right]\right) & \forall X \in \Gamma(T \mathcal{F}) \\ \pi\left(\nabla_{X}^{M} Y_{s}\right) & \forall X \in \Gamma\left(T \mathcal{F}^{\perp}\right)\end{cases}
$$

where $Y_{s} \in \Gamma\left(T \mathcal{F}^{\perp}\right)$ corresponding to $s$ under the canonical isomorphism $\sigma$. Let $R^{\nabla}$ and Ric $^{Q}$ be the curvature tensor and the transversal Ricci operator of $\mathcal{F}$, respectively. The mean curvature form $\kappa$ of $\mathcal{F}$ is given by

$$
\kappa(X)=g_{Q}\left(\sum_{i=1}^{p} \pi\left(\nabla_{E_{i}}^{M} E_{i}\right), \pi(X)\right) \quad \forall X \in \Gamma(T M),
$$

where $\left\{E_{i}\right\}_{i=1, \cdots, p}$ is a local orthonormal basis of $T \mathcal{F}$. Let $\Omega_{B}^{r}(\mathcal{F})$ be the space of all basic $r$-forms, i.e., $\phi \in \Omega_{B}^{r}(\mathcal{F})$ if and only if $\left.X\right\lrcorner \phi=0$ and $\mathcal{L}_{X} \phi=0$ for any $X \in T \mathcal{F}$, where $\left.X\right\lrcorner$ denotes the interior product. $\mathcal{F}$ is said to be minimal if $\kappa=0$. It is well-known([2]) that on a compact manifold, $\kappa_{B}$ is closed, i.e., $d \kappa_{B}=0$, where $\kappa_{B}$ is the basic component of $\kappa$ under the $L^{2}$-orthogonal decomposition $\Omega^{1}(M)=\Omega_{B}^{1}(\mathcal{F}) \oplus\left(\Omega_{B}^{1}(\mathcal{F})\right)^{\perp}([2]$, [31]). We note that the proofs of these facts in [31] are still valid on a manifold that is not necessarily compact if the leaf closures of the Riemannian foliation are compact. Now we recall the transversal star operator $\bar{*}: \Omega_{B}^{r}(\mathcal{F}) \rightarrow \Omega_{B}^{q-r}(\mathcal{F})$ given by

$$
\bar{*} \phi=(-1)^{p(q-r)} *\left(\phi \wedge \chi_{\mathcal{F}}\right),
$$

where $\chi_{\mathcal{F}}=E_{1}^{*} \wedge \ldots \wedge E_{p}^{*}$ is the characteristic form of $\mathcal{F}$ and $*$ is the Hodge star operator associated to $g_{M}$. Clearly, $\bar{*}^{2} \phi=(-1)^{r(q-r)} \phi$ for any $\left.\phi \in \Omega_{B}^{r} \mathcal{F}\right)$. Let $\nu$ be the transversal volume form; that is, $* \nu=\chi_{\mathcal{F}}$. Then the pointwise inner product $\langle\cdot, \cdot\rangle$ on $\Lambda^{r} Q^{*}$ is defined by $\langle\phi, \psi\rangle \nu=\phi \wedge \bar{*} \psi$. The global inner product on $L^{2}\left(\Omega_{B}^{r}(\mathcal{F})\right)$ is

$$
\ll \phi, \psi \gg=\int_{M}\langle\phi, \psi\rangle \mu_{M}
$$

where $\mu_{M}=\nu \wedge \chi_{\mathcal{F}}$ is the volume form with respect to $g_{M}$. Let

$$
d_{B}=\left.d\right|_{\Omega_{B}^{*}(\mathcal{F})}, \quad d_{T}=d_{B}-\epsilon\left(\kappa_{B}\right)
$$

where $\epsilon(\phi) \psi=\phi \wedge \psi$ for any $\phi \in \Omega_{B}^{1}(\mathcal{F})$. The operator $\epsilon$ acts pointwise and of course is define on $Q^{*} \cong\left(T \mathcal{F}^{\perp}\right)^{*}$. For $v \in Q \cong T \mathcal{F}^{\perp}$, let

$$
v\lrcorner=\left(\epsilon\left(v^{b}\right)\right)^{*}
$$

denote the interior product. 
Proposition 2.1. (In [2], [31] for the compact case) Let $(M, \mathcal{F})$ be a Riemannian foliation of codimension $q$ such that the leaf closures of $\mathcal{F}$ are compact. Then the formal adjoint operators $\delta_{B}$ and $\delta_{T}$ of $d_{B}$ and $d_{T}$ with respect to $\ll \cdot, \cdot \gg$ on basic forms are given by

$$
\left.\delta_{B} \phi=(-1)^{q(r+1)+1} \bar{*} d_{T} \bar{*} \phi=\delta_{T}+\kappa_{B}^{\sharp}\right\lrcorner, \quad \delta_{T} \phi=(-1)^{q(r+1)+1} \bar{*} d_{B} \bar{*} \phi,
$$

respectively, applied to basic $r$-forms $\phi$.

Proof. The proofs needed from [31] carry over to this slightly more general case.

Now we define two Laplacians $\Delta_{B}$ and $\Delta_{T}$ acting on $\Omega_{B}^{*}(\mathcal{F})$ by

$$
\Delta_{B}=d_{B} \delta_{B}+\delta_{B} d_{B}, \quad \Delta_{T}=d_{T} \delta_{T}+\delta_{T} d_{T},
$$

respectively. Then $\Delta_{T} \bar{*}=\bar{*} \Delta_{B}$. The first Laplacian $\Delta_{B}$ is said to be the basic Laplacian.

Now, we put $\mathcal{H}_{B}^{r}=\operatorname{ker} \Delta_{B}$ and $\mathcal{H}_{T}^{r}=\operatorname{ker} \Delta_{T}$. Then we have the following generalization of the usual De Rham-Hodge decompositon.

Theorem 2.2. ([2], [11], [24], [31]) Let $\left(M, g_{M}, \mathcal{F}\right)$ be a closed oriented manifold with a transversally oriented Riemannian foliation $\mathcal{F}$ and a bundle-like metric $g_{M}$. Then

$$
\begin{aligned}
\Omega_{B}^{r}(\mathcal{F}) & \cong \mathcal{H}_{B}^{r} \oplus \operatorname{Im} d_{B} \oplus \operatorname{Im} \delta_{B} \\
& \cong \mathcal{H}_{T}^{r} \oplus \operatorname{Im} d_{T} \oplus \operatorname{Im} \delta_{T}
\end{aligned}
$$

with finite dimensional $\mathcal{H}_{B}^{r}$ and $\mathcal{H}_{T}^{r}$.

Since $d_{B}^{2}=d_{T}^{2}=0$, we define basic cohomology groups $H_{B}^{r}$ and $H_{T}^{r}$ respectively by $H_{B}^{r}=\frac{\operatorname{ker} d_{B}}{\operatorname{Im} d_{B}}$ and $H_{T}^{r}=\frac{\operatorname{ker} d_{T}}{\operatorname{Im} d_{T}}$. Then $\mathcal{H}_{B}^{r} \cong H_{B}^{r}$ and $\mathcal{H}_{T}^{r} \cong H_{T}^{r}$ [33]. Since $\bar{*} \Delta_{B}=\Delta_{T} \bar{*}$, we have the twisted duality [23]

$$
\mathcal{H}_{B}^{r} \cong \mathcal{H}_{T}^{q-r} .
$$

Remark 2.3. Observe that, from the preceding results, the dimensions of $\mathcal{H}_{B}^{r}$ and $\mathcal{H}_{T}^{r}$ are smooth invariants of $\mathcal{F}$ and do not depend on the choices of bundle-like metric $g_{M}$ or on the transversal metric $g_{Q}$, even though the spaces of harmonic forms do depend on these choices.

If $\mathcal{F}$ is a taut foliation (i.e. $\kappa=0$ ), then Poincaré duality holds. That is, $\mathcal{H}_{B}^{r} \cong \mathcal{H}_{B}^{q-r}[22]$. Let $\left\{E_{a}\right\}$ and $\left\{\theta^{a}\right\}$ be the local orthonormal normal frame and its dual, respectively. Then we have the following generalized Weitzenböck formula.

Theorem 2.4. ([16, Formula 3.9] for the case of an isoparametric foliation) Let $\left(M, g_{M}, \mathcal{F}\right)$ be a Riemannian manifold with a foliation of codimension $q$ with compact leaf closures and a bundle-like metric $g_{M}$. Then the generalized Weitzenböck formula is given by the following: for any basic form $\phi \in \Omega_{B}^{r}(\mathcal{F})$,

$$
\Delta_{B} \phi=\nabla_{\mathrm{tr}}^{*} \nabla_{\mathrm{tr}} \phi+A_{\kappa_{B}^{\sharp}}(\phi)+F(\phi),
$$

where

$$
\begin{aligned}
& \nabla_{\mathrm{tr}}^{*} \nabla_{\mathrm{tr}} \phi=-\sum_{a} \nabla_{E_{a}} \nabla_{E_{a}} \phi+\nabla_{\kappa_{B}^{\sharp}} \phi, \\
& A_{Y}(\phi)=\mathcal{L}_{Y} \phi-\nabla_{Y} \phi, \quad Y \in T M, \\
& \left.F(\phi)=\sum_{a, b} \theta^{a} \wedge E_{b}\right\lrcorner R^{\nabla}\left(E_{b}, E_{a}\right) \phi .
\end{aligned}
$$

In particular, if $\phi$ is a basic 1-form, then $F(\phi)^{\sharp}=\operatorname{Ric}^{Q}\left(\phi^{\sharp}\right)$. 
It is well-known ([16, Proposition 3.1]) that the operator $\nabla_{\mathrm{tr}}^{*} \nabla_{\mathrm{tr}}$ is non-negative and formally self-adjoint on closed manifolds, that is,

$$
\int_{M}\left\langle\nabla_{\mathrm{tr}}^{*} \nabla_{\mathrm{tr}} \phi, \psi\right\rangle \mu_{M}=\int_{M}\left\langle\nabla_{\mathrm{tr}} \phi, \nabla_{\mathrm{tr}} \psi\right\rangle \mu_{M}
$$

for all $\phi, \psi \in \Omega_{B}^{r}(\mathcal{F})$, where $\left\langle\nabla_{\mathrm{tr}} \phi, \nabla_{\mathrm{tr}} \psi\right\rangle=\sum_{a=1}^{q}\left\langle\nabla_{E_{a}} \phi, \nabla_{E_{a}} \psi\right\rangle$.

Theorem 2.5. Let $\left(M, g_{M}, \mathcal{F}\right)$ be a closed Riemannian manifold with a foliation $\mathcal{F}$ of codimension $q$ and a bundle-like metric $g_{M}$. If the endomorphism $F$ is non-negative and positive at some point, then

$$
\mathcal{H}_{B}^{r}=\{0\} .
$$

Proof. Now, we modify the original bundle-like metric $g_{M}$ without changing the transversal metric $g_{Q}$ such that $\delta_{B} \kappa_{B}=0$. The existence of such metric is assured, from [26],[27]. With this new metric, the hypothesis on $F$ still holds, since this operator only depends on $g_{Q}$. Let $\phi$ be a basic-harmonic $r$-form. From (2.10), we have

$$
\int_{M}\left|\nabla_{\mathrm{tr}} \phi\right|^{2}+\int_{M}\langle F(\phi), \phi\rangle+\int_{M}\left\langle A_{\kappa_{B}^{\sharp}}(\phi), \phi\right\rangle=0 .
$$

Since $\left.A_{\kappa_{B}^{\sharp}}(\phi)=d_{B} \kappa_{B}^{\sharp}\right\lrcorner \phi-\nabla_{\kappa_{B}^{\sharp}} \phi$ and $\delta_{B} \phi=0$, we have

$$
\left.\int_{M}\left\langle A_{\kappa_{B}^{\sharp}}(\phi), \phi\right\rangle=\int_{M}\left\langle\kappa_{B}^{\sharp}\right\lrcorner \phi, \delta_{B} \phi\right\rangle+\frac{1}{2} \int_{M} \kappa_{B}^{\sharp}\left(|\phi|^{2}\right)=0 .
$$

Hence from (2.16), the proof is complete.

Let $\mathcal{R}^{\nabla}: \Lambda^{2} Q^{*} \rightarrow \Lambda^{2} Q^{*}$ be the transversal curvature operator, which is defined by

$$
\left\langle\mathcal{R}^{\nabla}\left(\omega_{1} \wedge \omega_{2}\right), \omega_{3} \wedge \omega_{4}\right\rangle=g_{Q}\left(R^{\nabla}\left(\omega_{1}^{\sharp}, \omega_{2}^{\sharp}\right) \omega_{4}^{\sharp}, \omega_{3}^{\sharp}\right),
$$

where $\omega_{i} \in Q^{*}(i=1, \cdots, 4)$. Then we recall a generalization of the Gallot-Meyer theorem for foliations.

Theorem 2.6. [21, Corollary 2.5] Let $\left(M, g_{M}, \mathcal{F}\right)$ be as in Theorem 2.4. If $\mathcal{R}^{\nabla} \geq C$ for a positive constant $C$, then

$$
\langle F(\phi), \phi\rangle \geq r(q-r) C|\phi|^{2}
$$

for any basic $r$-form $\phi(1 \leq r \leq q-1)$.

From Theorem 2.6, if the transversal curvature operator $\mathcal{R}^{\nabla}$ is positive-definite, then $F$ is also positive-definite. But the converse is not true. Hence we obtain the well-known vanishing theorem of basic-harmonic forms.

Corollary 2.7. [28, Corollary D] Let $\left(M, g_{M}, \mathcal{F}\right)$ be as in Theorem 2.5. If the transversal curvature operator is positive-definite, then $\mathcal{H}_{B}^{r}=0$ for $0<r<q$.

Now, we recall the transversal divergence theorem on a foliated manifold for later use.

Theorem 2.8. [36, Theorem A] Let $\left(M, g_{M}, \mathcal{F}\right)$ be a closed, oriented Riemannian manifold with a transversally oriented foliation $\mathcal{F}$ and a bundle-like metric $g_{M}$ with respect to $\mathcal{F}$. Then

$$
\int_{M} \operatorname{div}_{\nabla}(\pi(X)) \mu_{M}=\int_{M} g_{Q}\left(\pi(X), \kappa^{\sharp}\right) \mu_{M}
$$

for any $X \in \Gamma(T M)$, where $\operatorname{div}_{\nabla}(\pi(X))$ is the transversal divergence of $X$ with respect to $\nabla$. 


\section{The Basic Dolbeault COHOMOlOGY on TRAnSVERSEly KÄHLER FOliations}

In this section, we generally use the same notation and cite elementary results from [17, Section 3] and [30]. Let $\left(M, g_{M}, \mathcal{F}, J\right)$ be a Riemannian manifold with a transversely Kähler foliation $\mathcal{F}$ of codimension $q=2 n$. That is, $g_{M}$ is a bundle-like metric, and $J$ is a holonomyinvariant almost complex structure on $Q$ such that $\nabla g_{Q}=0$ and $\nabla J=0$, with $\nabla$ being the transversal Levi-Civita connection on $Q$, extended in the usual way to tensors [30]. In some of what follows, we will merely need the fact that the foliation is transversally Hermitian (all of the above, merely requiring $J$ is integrable and not that $\nabla J=0$ ), and other times we will need the full power of the Kähler condition $\nabla J=0$.

Note that for any $X, Y \in T M$,

$$
\omega(X, Y)=g_{Q}(\pi(X), J \pi(Y))
$$

defines a basic Kähler 2-form $\omega$, which is necessarily closed. Locally, the basic Kähler 2-form $\omega$ may be expressed by

$$
\omega=-\frac{1}{2} \sum_{\alpha=1}^{2 n} \theta^{\alpha} \wedge J \theta^{\alpha},
$$

where $\left\{\theta^{\alpha}\right\}_{\alpha=1, \cdots, 2 n}$ is a local orthonormal frame of $Q^{*}$. Here, we extend $J$ to elements of $Q^{*}$ by setting

$$
(J \theta)(X)=-\theta(J X)
$$

for any $X \in Q_{x}, \theta \in Q_{x}^{*}$. Note that if $J^{*}$ denotes the dual transformation on $Q^{*}$, the $J$ above satisfies $J^{*}=-J$. We prefer this convention so that $J$ commutes with the musical isomorphisms, so for example

$$
(J v)^{b}=J\left(v^{b}\right)
$$

for all $v \in Q$. When it is convenient, we will also refer to the bundle map $J^{\prime}: T M \rightarrow T M$ defined by $J^{\prime}(v)=J(\pi(v))$ and abuse notation by denoting $J=J^{\prime}$. Similarly, we sometimes will act on all of $T^{*} M$ using the symbol $J$. We note that all of the above is true for transversally Hermitian foliations, but the form $\omega$ is not closed unless the foliation is Kähler.

Example 3.1. Note that in contrast to the situation of a Kähler form on an ordinary manifold, it is possible that $\omega$ is a trivial class in basic cohomology. We consider the Carrière example from [7]. Let $A$ be a matrix in $\mathrm{SL}_{2}(\mathbb{Z})$ of trace strictly greater than 2 . We denote respectively by $V_{1}$ and $V_{2}$ unit eigenvectors associated with the eigenvalues $\lambda$ and $\frac{1}{\lambda}$ of $A$ with $\lambda>1$ irrational. Let the hyperbolic torus $\mathbb{T}_{A}^{3}$ be the quotient of $\mathbb{T}^{2} \times \mathbb{R}$ by the equivalence relation which identifies $(m, t)$ to $(A(m), t+1)$. The flow generated by the vector field $V_{2}$ is a Riemannian foliation with bundle-like metric (letting $(x, s, t)$ denote local coordinates in the $V_{2}$ direction, $V_{1}$ direction, and $\mathbb{R}$ direction, respectively)

$$
g=\lambda^{-2 t} d x^{2}+\lambda^{2 t} d s^{2}+d t^{2} .
$$

Note that the mean curvature of the flow is $\kappa=\kappa_{b}=\log (\lambda) d t$, since $\chi_{\mathcal{F}}=\lambda^{-t} d x$ is the characteristic form and $d \chi_{\mathcal{F}}=-\log (\lambda) \lambda^{-t} d t \wedge d x=-\kappa \wedge \chi_{\mathcal{F}}$. Then an orthonormal frame field for this manifold is $\left\{X=\lambda^{t} \partial_{x}, Y_{1}=\lambda^{-t} \partial_{s}, Y_{2}=\partial_{t}\right\}$ corresponding to the orthonormal coframe $\left\{X^{*}=\chi_{\mathcal{F}}=\lambda^{-t} d x, Y_{1}^{*}=\lambda^{t} d s, Y_{2}^{*}=d t\right\}$. Then, letting $J$ be defined by $J\left(Y_{1}\right)=$ $Y_{2}, J\left(Y_{2}\right)=-Y_{1}$, the Nijenhuis tensor

$$
N_{J}\left(Y_{1}, Y_{2}\right)=\left[Y_{1}, Y_{2}\right]+J\left(\left[J Y_{1}, Y_{2}\right]+\left[Y_{1}, J Y_{2}\right]\right)-\left[J Y_{1}, J Y_{2}\right]
$$

clearly vanishes, so that $J$ is integrable. 
The corresponding transverse Kähler form is seen to be $\omega=Y_{2}^{*} \wedge Y_{1}^{*}=\lambda^{t} d t \wedge d s=$ $d\left(\frac{1}{\log \lambda} Y_{1}^{*}\right)$, an exact form in basic cohomology.

Let $Q^{C}=Q \otimes \mathbb{C}$ be the complexified normal bundle and let

$$
Q^{1,0}=\left\{Z \in Q^{C} \mid J Z=i Z\right\}, \quad Q^{0,1}=\left\{Z \in Q^{C} \mid J Z=-i Z\right\} .
$$

The element of $Q^{1,0}$ (resp. $Q^{0,1}$ ) is called a complex normal vector field of type $(1,0)$ (resp. $(0,1))$. Then $Q^{C}=Q^{1,0} \oplus Q^{0,1}$ and

$$
Q^{1,0}=\{X-i J X \mid X \in Q\}, \quad Q^{0,1}=\{X+i J X \mid X \in Q\} .
$$

Now, let $Q_{C}^{*}$ be the real dual bundle of $Q^{C}$, defined at each $x \in M$ to be the $\mathbb{C}$-linear maps from $Q_{x}^{C}$ to $\mathbb{C}$. Let $\Lambda_{C} Q^{*}=\Lambda Q_{C}^{*}$. We define two sub-bundles $Q_{1,0}$ and $Q_{0,1}$ of $\Lambda_{C}^{1} Q^{*}$ by

$$
\begin{aligned}
& Q_{1,0}=\left\{\xi \in \Lambda_{C}^{1} Q^{*} \mid \xi(Z)=0, \forall Z \in Q^{0,1}\right\}, \\
& Q_{0,1}=\left\{\xi \in \Lambda_{C}^{1} Q^{*} \mid \xi(Z)=0, \forall Z \in Q^{1,0}\right\} .
\end{aligned}
$$

Then $\Lambda_{C}^{1} Q^{*}=Q_{1,0} \oplus Q_{0,1}$ and

$$
\begin{aligned}
& Q_{1,0}=\left\{\theta+i J \theta\left|\theta \in Q_{C}^{*}, \theta\right|_{Q} \in Q^{*}\right\} \quad \text { and } \\
& Q_{0,1}=\left\{\theta-i J \theta\left|\theta \in Q_{C}^{*}, \theta\right|_{Q} \in Q^{*}\right\},
\end{aligned}
$$

where $(J \theta)(X)=-\theta(J X)$ for any $X \in Q^{C}$. Let $\Lambda_{C}^{r, s} Q^{*}$ be the subspace of $\Lambda_{C} Q^{*}$ spanned by $\xi \wedge \eta$, where $\xi \in \Lambda^{r} Q_{1,0}$ and $\eta \in \Lambda^{s} Q_{0,1}$. The sections of $\Lambda_{C}^{r, s} Q^{*}$ are said to be forms of type $(r, s)$. Let $\Omega_{B}^{r, s}(\mathcal{F})$ be the set of the basic forms of type $(r, s)$. Let $\left\{E_{a}, J E_{a}\right\}_{a=1, \cdots, n}$ be a local orthonormal basic frame(called a J-basic frame) of $Q$ and $\left\{\theta^{a}, J \theta^{a}\right\}_{a=1, \cdots, n}$ be their dual basic forms on $Q^{*}$. Let $V_{a}=\frac{1}{\sqrt{2}}\left(E_{a}-i J E_{a}\right)$ and $\omega^{a}=\frac{1}{\sqrt{2}}\left(\theta^{a}+i J \theta^{a}\right)$. Then

$$
\omega^{a}\left(V_{b}\right)=\bar{\omega}^{a}\left(\bar{V}_{b}\right)=\delta_{a b}, \omega^{a}\left(\bar{V}_{b}\right)=\bar{\omega}^{a}\left(V_{b}\right)=0 .
$$

A frame field $\left\{V_{a}\right\}$ is a local orthonormal basic frame field on $Q^{1,0}$, which is called a normal frame field of type $(1,0)$, and $\left\{\omega^{a}\right\}$ is a dual coframe field on $Q_{1,0}$.

In what follows, we extend the connection $\nabla$ on $Q$ in the natural way so that $\nabla_{X} Y$ is defined for any $X \in \Gamma(T M \otimes \mathbb{C})$ and any $Y \in \Gamma\left(Q^{C}\right)$. We further extend it to differential forms, requiring that $\nabla$ is a Hermitian connection, i.e., for any $V \in Q^{C}$ and any $\phi, \psi \in$ $\Omega_{B}^{r, s}(\mathcal{F})$

$$
V\langle\phi, \psi\rangle=\left\langle\nabla_{V} \phi, \psi\right\rangle+\left\langle\phi, \nabla_{\bar{V}} \psi\right\rangle
$$

It is an easy exercise to show that for any complex vector field $X, \nabla_{X}$ preserves the $(r, s)$ type of the form or vector field.

Definition 3.2. Let $\left(M, g_{Q}, \mathcal{F}, J\right)$ be a transversely holomorphic foliation. Then a complex normal vector field $Z$ of type $(1,0)$ is said to be transversally holomorphic if

$$
\nabla_{\bar{V}_{a}} Z=0 \text { for } 1 \leq a \leq n .
$$

Let $V(\mathcal{F})$ be the set of infinitesimal automorphisms $X$, i.e., $[X, Y] \in T \mathcal{F}$ for any $Y \in T \mathcal{F}$. The Lie derivative $\mathcal{L}_{X}$ can be applied to tensors with inputs in $Q$ or $Q^{*}$, because all such tensors can be extended by 0 to tensors with inputs in $T M$ and $T^{*} M$. We will now consider what is true when the Kähler condition $\nabla J=0$ holds. 
Proposition 3.3. Let $\left(M, g_{Q}, \mathcal{F}, J\right)$ be a transversely Kähler foliation with compact leaf closures, with bundle-like metric $g_{M}$ and $X \in V(\mathcal{F})$. Then the following are equivalent.

(1) A vector field $\pi(X)$ is transversally automorphic, i.e., $\mathcal{L}_{X} J=0$ (if and only if $\nabla_{J Y} \pi(X)=J \nabla_{Y} \pi(X)$ for any $\left.Y \in T \mathcal{F}^{\perp}\right)$.

(2) A complex normal vector field $Z=\pi(X)-i J \pi(X)$ is transversally holomorphic, i.e., $\nabla_{\bar{V}_{a}} Z=0$ for $1 \leq a \leq n$.

Now we recall the following proposition.

Proposition 3.4. [18, Theorem 5.2] and [17, Corollary 5.10] Let $\left(M, g_{Q}, \mathcal{F}, J\right)$ be a transversely Kähler foliation on a compact Riemannian manifold with bundle-like metric $g_{M}$. Then for any $X \in V(\mathcal{F})$,

(1) $\pi(X)$ is transversally automorphic if and only if

$$
\begin{gathered}
\nabla_{\mathrm{tr}}^{*} \nabla_{\mathrm{tr}} \pi(X)-\operatorname{Ric}^{Q}(X)+A_{X} \kappa_{B}^{\sharp}=0, \\
\int_{M} g_{Q}\left(\left(\mathcal{L}_{X} J\right) \kappa_{B}^{\sharp}, J \pi(X)\right)=0 .
\end{gathered}
$$

(2) $\pi(X)$ is transversally Killing (i.e., $\left.\mathcal{L}_{X} g_{Q}=0\right)$ if and only if

$$
\nabla_{\mathrm{tr}}^{*} \nabla_{\mathrm{tr}} \pi(X)-\operatorname{Ric}^{Q}(X)+A_{X} \kappa_{B}^{\sharp}=0, \quad \operatorname{div}_{\nabla}(\pi(X))=0 .
$$

Remark 3.5. Let $\left(M, g_{Q}, \mathcal{F}, J\right)$ be a transversely Kähler foliation on a compact Riemannian manifold with bundle-like metric $g_{M}$. Then from [17, Theorem 5.8, Corollaries 5.9 and 5.10]

(1) If $\mathcal{F}$ is minimal, then every transversal Killing field is transversally automorphic.

(2) If $\mathcal{F}$ is non-minimal and the transversal Ricci curvature is positive, then every transversal Killing field is transversally automorphic.

Now, let $\langle\cdot, \cdot\rangle$ be a Hermitian inner product on $\Lambda_{C}^{r, s}(\mathcal{F})$ induced by the metric, and let $\bar{*}: \Lambda_{C}^{r, s}(\mathcal{F}) \rightarrow \Lambda_{C}^{n-s, n-r}(\mathcal{F})$ be the star operator defined by

$$
\phi \wedge \bar{*} \bar{\psi}=\langle\phi, \psi\rangle \nu
$$

where $\nu=\frac{\omega^{n}}{n !}$ is the transverse volume form.

Then for any $\psi \in \Lambda_{C}^{r, s}(\mathcal{F})$,

$$
\bar{*} \psi=\bar{*} \bar{\psi}, \quad \bar{*}^{2} \psi=(-1)^{r+s} \psi .
$$

We define the operators $\partial_{B}: \Omega_{B}^{r, s}(\mathcal{F}) \rightarrow \Omega_{B}^{r+1, s}(\mathcal{F})$ and $\bar{\partial}_{B}: \Omega_{B}^{r, s}(\mathcal{F}) \rightarrow \Omega_{B}^{r, s+1}(\mathcal{F})$ in the usual way using the differential $d=\partial_{B}+\bar{\partial}_{B}$ and the projections $\Omega_{B} \rightarrow \Omega_{B}^{r, s}$. Because of the given transverse Hermitian structure, the complex differentials satisfy

$$
\begin{aligned}
& \partial_{B} \phi=\sum_{a=1}^{n} \omega^{a} \wedge \nabla_{V_{a}} \phi, \\
& \bar{\partial}_{B} \phi=\sum_{a=1}^{n} \bar{\omega}^{a} \wedge \nabla_{\bar{V}_{a}} \phi,
\end{aligned}
$$

respectively. 
We now write

$$
\begin{aligned}
\kappa_{B} & =\kappa_{B}^{1,0}+\kappa_{B}^{0,1} \\
\kappa_{B}^{1,0} & =\frac{1}{2}\left(\kappa_{B}+i J \kappa_{B}\right) \in \Omega_{B}^{1,0}(\mathcal{F}) \\
\kappa_{B}^{0,1} & =\frac{\kappa_{B}^{1,0}}{2}=\frac{1}{2}\left(\kappa_{B}-i J \kappa_{B}\right) \in \Omega_{B}^{0,1}(\mathcal{F})
\end{aligned}
$$

Now we utilize the bundle-like metric $g_{M}$ to define the operators $\partial_{T}: \Omega_{B}^{r, s}(\mathcal{F}) \rightarrow \Omega_{B}^{r+1, s}(\mathcal{F})$ and $\bar{\partial}_{T}: \Omega_{B}^{r, s}(\mathcal{F}) \rightarrow \Omega_{B}^{r, s+1}(\mathcal{F})$ respectively by

$$
\partial_{T} \phi=\partial_{B} \phi-\epsilon\left(\kappa_{B}^{1,0}\right) \phi, \quad \bar{\partial}_{T} \phi=\bar{\partial}_{B} \phi-\epsilon\left(\kappa_{B}^{0,1}\right) \phi
$$

Then by (2.5) we have

$$
d_{T}=\partial_{T}+\bar{\partial}_{T}
$$

From Proposition 2.1, the adjoint operators $\delta_{B}$ and $\delta_{T}$ of $d_{B}$ and $d_{T}$ are given by

$$
\delta_{B}=-\bar{*} d_{T} \bar{*}, \quad \delta_{T}=-\bar{*} d_{B} \bar{*},
$$

respectively.

Let $\partial_{T}^{*}, \bar{\partial}_{T}^{*}, \partial_{B}^{*}$ and $\bar{\partial}_{B}^{*}$ be the formal adjoint operators of $\partial_{T}, \bar{\partial}_{T}, \partial_{B}$ and $\bar{\partial}_{B}$, respectively, on the space of basic forms. Then

$$
\delta_{B}=\partial_{B}^{*}+\bar{\partial}_{B}^{*}, \quad \delta_{T}=\partial_{T}^{*}+\bar{\partial}_{T}^{*}
$$

and from (3.14),

$$
\begin{array}{ll}
\partial_{T}^{*} \phi=-\bar{*} \bar{\partial}_{B} \bar{*} \phi, & \bar{\partial}_{T}^{*} \phi=-\bar{*} \partial_{B} \bar{*} \phi, \\
\partial_{B}^{*} \phi=-\bar{*} \bar{\partial}_{T} \bar{*} \phi, & \bar{\partial}_{B}^{*} \phi=-\bar{*} \partial_{T} \bar{*} \phi .
\end{array}
$$

These formulas were first used in the case of minimalizable (homologically oriented) transverse Kähler foliations in [10, Section 3.4]. In what follows, we let

$$
H^{1,0}=\overline{\left(\kappa_{B}^{1,0}\right)^{\sharp}}=\frac{1}{2}\left(\kappa_{B}^{\sharp}-i J \kappa_{B}^{\sharp}\right), \quad H^{0,1}=\overline{H^{1,0}} .
$$

We will extend the definitions of exterior product $\epsilon$ and interior product $\lrcorner$ linearly to complex vectors. Observe that $V\lrcorner$ is by definition the adjoint of $\epsilon\left(V^{b}\right)$ on real vector fields, but on complex vector fields an adjustment must be made. If $v, w$ are real tangent vectors, the interior product satisfies

$$
\begin{aligned}
(v-i w)\lrcorner & =(v\lrcorner)-i(w\lrcorner)=\left(\epsilon\left(v^{b}\right)-i \epsilon\left(w^{b}\right)\right)^{*} \\
& =\left(\epsilon\left(v^{b}-i w^{b}\right)\right)^{*} .
\end{aligned}
$$

So if we denote $X^{b}=v^{b}+i w^{b}$ for any $X=v+i w$, then for complex vectors $X$,

$$
\left.(X\lrcorner)^{*}=\epsilon\left(X^{b}\right), \quad\left(\epsilon\left(X^{b}\right)\right)^{*}=X\right\lrcorner .
$$

Then for complex vectors $X$ it follows that

$$
\left.\left.\bar{*} \epsilon\left(X^{b}\right) \bar{*}=X\right\lrcorner, \quad \bar{*}(X\lrcorner\right) \bar{*}=-\epsilon\left(X^{b}\right)
$$

Then we have the following. 
Proposition 3.6. (Can be deduced from [17, Section 3] and [19, Theorem 3.1] in transversely Kähler case) If $\left(M, g_{Q}, \mathcal{F}, J\right)$ is a transversely Hermitian foliation with compact leaf closures and bundle-like metric $g_{M}$, we have

$$
\left.\left.\partial_{B}^{*} \phi=\partial_{T}^{*} \phi+H^{1,0}\right\lrcorner \phi, \quad \bar{\partial}_{B}^{*} \phi=\bar{\partial}_{T}^{*} \phi+H^{0,1}\right\lrcorner \phi,
$$

where

$$
\left.\left.\partial_{T}^{*} \phi=-\sum_{a=1}^{n} V_{a}\right\lrcorner \nabla_{\bar{V}_{a}} \phi, \quad \bar{\partial}_{T}^{*} \phi=-\sum_{a=1}^{n} \bar{V}_{a}\right\lrcorner \nabla_{V_{a}} \phi .
$$

Proof. Observe that we just apply the operators $\delta_{B}^{*}$ and $\delta_{T}^{*}$ to forms of type $(r, s)$ and take the $(r-1, s)$ and $(r, s-1)$ components of the result, using the standard formulas for the divergence and Proposition 2.1.

We note that similar calculations were done for the twisted Dolbeault operator in [14, Section 3].

We now assume that our foliation is transversally Kähler. Let $L: \Omega_{B}^{r}(\mathcal{F}) \rightarrow \Omega_{B}^{r+2}(\mathcal{F})$ and $\Lambda: \Omega_{B}^{r}(\mathcal{F}) \rightarrow \Omega_{B}^{r-2}(\mathcal{F})$ be given by

$$
L(\phi)=\omega \wedge \phi, \quad \Lambda(\phi)=\omega\lrcorner \phi,
$$

respectively, where $\left.\left.\left.\left(\xi_{1} \wedge \xi_{2}\right)\right\lrcorner=\xi_{2}^{\sharp}\right\lrcorner \xi_{1}^{\sharp}\right\lrcorner$ for any basic 1 -forms $\xi_{i}(i=1,2)$. We have $\langle L \phi, \psi\rangle=$ $\langle\phi, \Lambda \psi\rangle$ and $\Lambda=(-1)^{j} \bar{*} \bar{*}$ on basic $j$-forms. For any real vector $X \in Q$, we have [17] that

$$
\begin{aligned}
& \left.[L, X\lrcorner]=\epsilon\left(J X^{b}\right), \quad\left[\Lambda, \epsilon\left(X^{b}\right)\right]=-(J X)\right\lrcorner, \\
& \left.\left[L, \epsilon\left(X^{b}\right)\right]=[\Lambda, X\lrcorner\right]=0 .
\end{aligned}
$$

A simple calculation shows that the formulas above extend to complex vectors $X$ as well. Now, we extend the complex structure $J$ to $\Omega_{B}^{r}(\mathcal{F})$ by

$$
\left.J \phi=\sum_{a=1}^{2 n} J \theta^{a} \wedge E_{a}\right\lrcorner \phi .
$$

Then this formula is consistent with (3.3) $, J: \Omega_{B}^{r, s}(\mathcal{F}) \rightarrow \Omega_{B}^{r, s}(\mathcal{F})$ is skew-Hermitian, i.e., $\langle J \phi, \psi\rangle+\langle\phi, J \psi\rangle=0$, and $J \phi=i(s-r) \phi$ for any $\phi \in \Omega_{B}^{r, s}(\mathcal{F})$. In addition $(J \alpha)^{\sharp}=J\left(\alpha^{\sharp}\right)$ for any one-form $\alpha$. Note that this $J$ is much different from the operator $C$ induced from the pullback $J^{*}$ used often in Kähler geometry.

Proposition 3.7. [17, Proposition 3.3] If $\left(M, g_{Q}, \mathcal{F}, J\right)$ is a transversely Kähler foliation with compact leaf closures and a bundle-like metric $g_{M}$, we have

$$
[L, J]=[\Lambda, J]=\left[L, d_{B}\right]=\left[\Lambda, \delta_{B}\right]=0 .
$$

Then we have the following corollary.

Corollary 3.8. [17, Proposition 3.4] With the same hypothesis, we have

$$
\begin{aligned}
& {\left[L, \partial_{B}\right]=\left[L, \bar{\partial}_{B}\right]=\left[\Lambda, \partial_{B}^{*}\right]=\left[\Lambda, \bar{\partial}_{B}^{*}\right]=0} \\
& {\left[L, \partial_{B}^{*}\right]=-i \bar{\partial}_{T},\left[L, \bar{\partial}_{B}^{*}\right]=i \partial_{T},\left[\Lambda, \partial_{B}\right]=-i \bar{\partial}_{T}^{*},\left[\Lambda, \bar{\partial}_{B}\right]=i \partial_{T}^{*} .}
\end{aligned}
$$

Remark 3.9. All equations in Proposition 3.7 and Corollary 3.8 hold if we exchange the operators $(\cdot)_{B}$ and $(\cdot)_{T}$. These results were shown in the minimal foliation case, when $(\cdot)_{B}=$ $(\cdot)_{T}$, in [10, Lemma 3.4.4]. 
Let $\square_{B}$ and $\bar{\square}_{B}$ be Laplace operators, which are defined by

$$
\square_{B}=\partial_{B} \partial_{B}^{*}+\partial_{B}^{*} \partial_{B} \quad \text { and } \quad \bar{\square}_{B}=\bar{\partial}_{B} \bar{\partial}_{B}^{*}+\bar{\partial}_{B}^{*} \bar{\partial}_{B},
$$

respectively. Clearly, $\square_{B}$ and $\square_{B}$ preserve the types of the forms.

Lemma 3.10. Let $\left(M, g_{Q}, \mathcal{F}, J\right)$ be a transversely Kähler foliation with compact leaf closures and bundle-like metric $g_{M}$. Then

$$
\begin{aligned}
\square_{B} & \left.\left.\left.\left.=\bar{\square}_{B}+\partial_{B} H^{1,0}\right\lrcorner+H^{1,0}\right\lrcorner \partial_{B}-\bar{\partial}_{B} H^{0,1}\right\lrcorner-H^{0,1}\right\lrcorner \bar{\partial}_{B}, \\
\Delta_{B} & \left.\left.\left.\left.=\square_{B}+\bar{\square}_{B}+\partial_{B} H^{0,1}\right\lrcorner+H^{0,1}\right\lrcorner \partial_{B}+\bar{\partial}_{B} H^{1,0}\right\lrcorner+H^{1,0}\right\lrcorner \bar{\partial}_{B} . \\
0 & =\bar{\partial}_{T}^{*} \bar{\partial}_{B}+\bar{\partial}_{B} \bar{\partial}_{T}^{*}-\partial_{T}^{*} \partial_{B}-\partial_{B} \partial_{T}^{*}
\end{aligned}
$$

If $\mathcal{F}$ is minimal, then

$$
\Delta_{B}=2 \square_{B}=2 \bar{\square}_{B}
$$

Proof. Since $d_{B}^{2}=d_{T}^{2}=0, \partial_{B}^{2}=\bar{\partial}_{B}^{2}=\partial_{B} \bar{\partial}_{B}+\bar{\partial}_{B} \partial_{B}=0$ and $\partial_{T}^{2}=\bar{\partial}_{T}^{2}=\partial_{T} \bar{\partial}_{T}+\bar{\partial}_{T} \partial_{T}=0$. From the equations above, Proposition 3.6 and Corollary 3.8 , we have

$$
\begin{aligned}
i\left(\partial_{B} \partial_{B}^{*}+\partial_{B}^{*} \partial_{B}\right) & =\partial_{B} \Lambda \bar{\partial}_{T}+\Lambda \bar{\partial}_{T} \partial_{B}-\partial_{B} \bar{\partial}_{T} \Lambda-\bar{\partial}_{T} \Lambda \partial_{B} \\
& =\left[\partial_{B}, \Lambda\right] \bar{\partial}_{B}+\bar{\partial}_{B}\left[\partial_{B}, \Lambda\right]+\partial_{B}\left[\epsilon\left(\kappa_{B}^{0,1}\right), \Lambda\right]+\left[\epsilon\left(\kappa_{B}^{0,1}\right), \Lambda\right] \partial_{B} \\
& =i\left(\bar{\partial}_{T}^{*} \bar{\partial}_{B}+\bar{\partial}_{B} \bar{\partial}_{T}^{*}\right)+\partial_{B}\left[\epsilon\left(\kappa_{B}^{0,1}\right), \Lambda\right]+\left[\epsilon\left(\kappa_{B}^{0,1}\right), \Lambda\right] \partial_{B} .
\end{aligned}
$$

Similarly, we have

$$
\begin{aligned}
& i\left(\bar{\partial}_{B} \partial_{B}^{*}+\partial_{B}^{*} \bar{\partial}_{B}\right)=\bar{\partial}_{B}\left[\epsilon\left(\kappa_{B}^{0,1}\right), \Lambda\right]+\left[\epsilon\left(\kappa_{B}^{0,1}\right), \Lambda\right] \bar{\partial}_{B} \\
& i\left(\partial_{B} \bar{\partial}_{B}^{*}+\bar{\partial}_{B}^{*} \partial_{B}\right)=-\partial_{B}\left[\epsilon\left(\kappa_{B}^{1,0}\right), \Lambda\right]-\left[\epsilon\left(\kappa_{B}^{1,0}\right), \Lambda\right] \partial_{B} .
\end{aligned}
$$

From (3.24) and (3.18), we have $\left.\left[\epsilon\left(\kappa_{B}^{0,1}\right), \Lambda\right]=i H^{1,0}\right\lrcorner$ and $\left.\left[\epsilon\left(\kappa_{B}^{1,0}\right), \Lambda\right]=-i H^{0,1}\right\lrcorner$. Hence (3.30) is proved from (3.34) using Proposition 3.6. By direct calculation, we have

$$
\Delta_{B}=\square_{B}+\bar{\square}_{B}+\left(\bar{\partial}_{B} \partial_{B}^{*}+\partial_{B}^{*} \bar{\partial}_{B}\right)+\left(\partial_{B} \bar{\partial}_{B}^{*}+\bar{\partial}_{B}^{*} \partial_{B}\right) .
$$

From (3.35) and (3.36), the proof of (3.31) is complete. Formula (3.32) follows from adding (3.34) to its conjugate and comparing with (3.30).

The part of the Lemma above for the minimal (homologically orientable) foliation case was first shown in [10, Proposition 3.4.5]. In that case, a consequence [10, Théorème 3.4.6] is that $\mathcal{H}_{B}^{k}=\bigoplus_{r+s=k} \mathcal{H}_{B}^{r, s}$ for all $k$, where $\mathcal{H}_{B}^{r, s}=\operatorname{ker} \bar{\square}_{B}$, which is in general false.

Corollary 3.11. With the hypothesis of Lemma 3.10:

If $\phi$ is of type $(r, 0)$, then

$$
\left.\Delta_{B} \phi=2 \bar{\square}_{B} \phi+\mathcal{L}_{H^{1,0}} \phi-H^{0,1}\right\lrcorner \bar{\partial}_{B} \phi .
$$

If $\phi$ is of type $(r, n)$, then

$$
\left.\left.\left.\Delta_{B} \phi=2 \bar{\square}_{B} \phi+\partial_{B} \kappa_{B}^{\sharp}\right\lrcorner \phi+\kappa_{B}^{\sharp}\right\lrcorner \partial_{B} \phi-\bar{\partial}_{B} H^{0,1}\right\lrcorner \phi .
$$

If $\phi$ is of type $(0, s)$, then

$$
\left.\left.\left.\Delta_{B} \phi=2 \bar{\square}_{B} \phi+\left(\partial_{B}-\bar{\partial}_{B}\right) H^{0,1}\right\lrcorner \phi+H^{0,1}\right\lrcorner\left(\partial_{B}-\bar{\partial}_{B}\right) \phi+H^{1,0}\right\lrcorner \partial_{B} \phi .
$$

If $\phi$ is of type $(n, s)$, then

$$
\left.\left.\left.\Delta_{B} \phi=2 \bar{\square}_{B} \phi-i\left(J \kappa_{B}^{\sharp}\right)\right\lrcorner \bar{\partial}_{B} \phi-i \bar{\partial}_{B}\left(J \kappa_{B}^{\sharp}\right)\right\lrcorner \phi+\partial_{B} H^{1,0}\right\lrcorner \phi .
$$


Proof. Let $\phi$ be a type $(r, 0)$. Then $\left.\left.H^{0,1}\right\lrcorner \phi=H^{0,1}\right\lrcorner \partial_{B} \phi=0$. From (3.30) and (3.31) and Cartan's formula for the Lie derivative, the proof of (3.38) is completed. The others are similarly proved.

Corollary 3.12. Let $\phi$ be a basic form of type $(r, s)$. Then $\phi$ is basic-harmonic if and only if $\left(\square_{B}+\square_{B}\right) \phi=0$ and

$$
\left.\left.\left.\left.\left\{H^{0,1}\right\lrcorner \partial_{B}+\partial_{B} H^{0,1}\right\lrcorner\right\} \phi=\left\{H^{1,0}\right\lrcorner \bar{\partial}_{B}+\bar{\partial}_{B} H^{1,0}\right\lrcorner\right\} \phi=0 .
$$

Proof. Let $\phi \in \Omega_{B}^{r, s}(\mathcal{F})$ be a basic form of type $(r, s)$. Clearly, $\left(\square_{B}+\bar{\square}_{B}\right) \phi \in \Omega_{B}^{r, s}(\mathcal{F})$, $\left.\left.\left\{H^{0,1}\right\lrcorner \partial_{B}+\partial_{B} H^{0,1}\right\lrcorner\right\} \phi \in \Omega_{B}^{r+1, s-1}(\mathcal{F})$ and $\left.\left.\left\{H^{1,0}\right\lrcorner \bar{\partial}_{B}+\bar{\partial}_{B} H^{1,0}\right\lrcorner\right\} \phi \in \Omega_{B}^{r-1, s+1}(\mathcal{F})$. Hence from (3.31), the proof follows.

Corollary 3.13. Let $\left(M, g_{Q}, \mathcal{F}, J\right)$ be a transversely Kähler foliation with compact leaf closures and a bundle-like metric $g_{M}$. Then the Laplacian on complex-valued functions satisfies

$$
\begin{aligned}
\square_{B} & =\bar{\square}_{B}-i\left(J \kappa_{B}\right)^{\sharp}, \\
\Delta_{B} & =\square_{B}+\bar{\square}_{B}, \\
\bar{\partial}_{T}^{*} \bar{\partial}_{B} & =\partial_{T}^{*} \partial_{B} .
\end{aligned}
$$

The second equation holds more generally for all transversely Hermitian foliations.

Proof. Equation (3.43) follows from (3.30), using $\left.\left.H^{1,0}\right\lrcorner \partial_{B} f=H^{1,0}\right\lrcorner\left(\partial_{B}+\bar{\partial}_{B}\right) f=$ $\left.H^{1,0}\right\lrcorner d_{B} f=H^{1,0} f$ for all complex-valued functions $f$. Equation (3.44) comes from expanding $\Delta_{B}=d_{B} \delta_{B}+\delta_{B} d_{B}$ with $d_{B}=\partial_{B}+\bar{\partial}_{B}$. Equation (3.45) follows from (3.32).

Corollary 3.14. Let $\left(M, g_{Q}, \mathcal{F}, J\right)$ be a transversely Kähler foliation with compact leaf closures and a bundle-like metric $g_{M}$. Then the Laplacian on complex-valued functions satisfies $\Delta_{B}=2 \bar{\square}_{B}$ if and only if $\kappa_{B}=0$.

Lemma 3.15. Let $(M, \mathcal{F}, J)$ be a transversely Hermitian foliation. Then a complex vector field $Z \in Q^{1,0}$ of type $(1,0)$ is transversally holomorphic (equivalently, $Z+\bar{Z}$ is transversally automorphic) if and only if

$$
\left.\left.\bar{\partial}_{B} Z\right\lrcorner+Z\right\lrcorner \bar{\partial}_{B}=0 .
$$

Proof. Let $Z \in Q^{1,0}$ be a transversally holomorphic vector field, that is, $\nabla_{\bar{V}_{a}} Z=0$ $(1 \leq a \leq n)$. Then for any $\phi \in \Omega_{B}^{r, s}(\mathcal{F})$,

$$
\begin{aligned}
\left.\bar{\partial}_{B} Z\right\lrcorner \phi & \left.\left.=\sum_{a} \bar{\omega}^{a} \wedge\left(\nabla_{\bar{V}_{a}} Z\right)\right\lrcorner \phi+\sum_{a} \bar{\omega}^{a} \wedge Z\right\lrcorner \nabla_{\bar{V}_{a}} \phi \\
& =-Z\lrcorner \bar{\partial}_{B} \phi,
\end{aligned}
$$

which proves (3.46). Conversely, if $Z$ satisfies (3.46), then

$$
\begin{aligned}
0 & \left.\left.=\bar{\partial}_{B} Z\right\lrcorner \omega^{b}+Z\right\lrcorner \bar{\partial}_{B} \omega^{b} \\
& \left.\left.=\sum_{a} \bar{\omega}^{a} \wedge \nabla_{\bar{V}_{a}}(Z\lrcorner \omega^{b}\right)-\sum_{a} \bar{\omega}^{a} \wedge Z\right\lrcorner \nabla_{\bar{V}_{a}} \omega^{b} \\
& \left.=\sum_{a}\left(\nabla_{\bar{V}_{a}} Z\right\lrcorner \omega^{b}\right) \bar{\omega}^{a} .
\end{aligned}
$$

Hence $\left.\nabla_{\bar{V}_{a}} Z\right\lrcorner \omega^{b}=0$ for any $a$ and $b$, which means $\nabla_{\bar{V}_{a}} Z=0$ for any $a$. That is, $Z$ is transversally holomorphic. 
Definition 3.16. On a transversely holomorphic foliation, a basic form $\phi$ is said to be basic holomorphic if $\bar{\partial}_{B} \phi=0$.

Proposition 3.17. Let $\left(M, g_{Q}, \mathcal{F}, J\right)$ be a transversely Kähler foliation with compact leaf closures and a bundle-like metric $g_{M}$, such that $\kappa_{B}^{\sharp}$ is transversally automorphic. If $\phi$ is a basic holomorphic form of type $(r, 0)$, then $\square_{B} \phi=\Delta_{B} \phi$ and $\left(\partial_{B} \partial_{T}^{*}+\partial_{T}^{*} \partial_{B}\right) \phi=0$.

Proof. Let $\phi$ be a basic holomorphic form of type $(r, 0)$, i.e., $\bar{\partial}_{B} \phi=0$. Moreover, $\bar{\partial}_{B}^{*} \phi=0$ because $\bar{\partial}_{B}^{*} \phi$ is of type $(r,-1)$. Hence $\bar{\square}_{B} \phi=0$. Since $\kappa_{B}^{\sharp}$ is transversally automorphic, from Lemma 3.15

$$
\left.\bar{\partial}_{B} H^{1,0}\right\lrcorner \phi=0 .
$$

That is, $\left.H^{1,0}\right\lrcorner \phi$ is a basic holomorphic form of type $(r-1,0)$. On the other hand,

$$
\left.\left.H^{0,1}\right\lrcorner \phi=H^{0,1}\right\lrcorner \partial_{B} \phi=0,
$$

because $\left.H^{0,1}\right\lrcorner \phi$ is of type $(r,-1)$ and $\left.H^{0,1}\right\lrcorner \partial_{B} \phi$ is of type $(r+1,-1)$. Hence from (3.31), we have

$$
\square_{B} \phi=\Delta_{B} \phi
$$

And from (3.32), $\left(\partial_{B} \partial_{T}^{*}+\partial_{T}^{*} \partial_{B}\right) \phi=0$ is proved.

The following example shows that in general we do not expect the mean curvature to be transversally automorphic for any metric.

Example 3.18. We continue from Example 3.1. From previous calculations,

$$
\begin{aligned}
\kappa & =\kappa_{B}=\log (\lambda) d t=\log (\lambda) Y_{2}^{*} \\
Y_{1}^{*} & =\lambda^{t} d s, Z^{*}=\frac{1}{2}\left(Y_{1}^{*}+i Y_{2}^{*}\right),
\end{aligned}
$$

so

$$
\begin{aligned}
\kappa_{B} & =-i(\log \lambda) Z^{*}+i(\log \lambda) \bar{Z}^{*} \\
& =-i(\log \lambda) \frac{1}{2}\left(\lambda^{t} d s+i d t\right)+i \frac{1}{2}(\log \lambda) \bar{Z}^{*}
\end{aligned}
$$

Then

$$
\begin{aligned}
\kappa_{B}^{1,0} & =-i(\log \lambda) Z^{*}=-\frac{i}{2}(\log \lambda)\left(\lambda^{t} d s+i d t\right) \\
\bar{\partial}_{B} \kappa_{B}^{1,0} & =d \kappa_{B}^{1,0}=\frac{i}{2}(\log \lambda)^{2} \lambda^{t} d s \wedge d t \\
& =\frac{i}{2}(\log \lambda)^{2} Y_{1}^{*} \wedge Y_{2}^{*} \\
& =(\log \lambda)^{2} \bar{Z}^{*} \wedge Z^{*}
\end{aligned}
$$

It is impossible to change the metric so that this is zero. The reason is that from [2] the mean curvature $\kappa_{B}^{\prime}$ for any other compatible bundle-like metric would satisfy $\kappa_{B}^{\prime}=\kappa_{B}+d f$ for some real basic function $f$, which would imply that $\left(\kappa_{B}^{1,0}\right)^{\prime}=\kappa_{B}^{1,0}+\partial_{B} f$, and $\partial_{B} f=Z(f) Z^{*}$. Since 
$f$ is a periodic function of $t$ alone, this is $\partial_{B} f=-\frac{i}{2}\left(\partial_{t} f\right) Z^{*}$. Then in that case

$$
\begin{aligned}
\bar{\partial}_{B}\left(\kappa_{B}^{1,0}\right)^{\prime} & =d\left(\kappa_{B}^{1,0}-\frac{i}{2}\left(\partial_{t} f\right) Z^{*}\right) \\
& =d\left(-\frac{i}{2}\left(\log \lambda+\frac{1}{2} \partial_{t} f\right)\left(\lambda^{t} d s+i d t\right)\right) \\
& =\left(\frac{i}{2}(\log \lambda)^{2}+\frac{i}{4}\left(\partial_{t}^{2} f+(\log \lambda) \partial_{t} f\right)\right) \lambda^{t} d s \wedge d t \\
& =\left((\log \lambda)^{2}+\frac{1}{2}\left(\partial_{t}^{2} f+(\log \lambda) \partial_{t} f\right)\right) \bar{Z}^{*} \wedge Z^{*}
\end{aligned}
$$

Since the term in parentheses is never zero for any periodic function $f$, we conclude that $\bar{\partial} \kappa_{B}^{1,0}$ is a nonzero multiple of $\bar{Z}^{*} \wedge Z^{*}$ for any compatible bundle-like metric. Further, we see that $\bar{\partial}_{B}=\bar{Z}^{*} \wedge \nabla_{\bar{Z}}$, so that

$$
\nabla_{\bar{Z}} \kappa_{B}^{1,0}=(\log \lambda)^{2} Z^{*}
$$

and likewise this would be nonzero for any compatible bundle-like metric. Hence $\kappa_{B}^{\sharp}$ is not automorphic in general for nontaut transversely Kähler foliations.

Proposition 3.19. Let $\left(M, g_{Q}, \mathcal{F}, J\right)$ be a transversely Kähler foliation with compact leaf closures and a bundle-like metric $g_{M}$. Let $\phi$ be a basic holomorphic form of type $(r, 0)$. Then

$$
\Delta_{B} \phi=\mathcal{L}_{H^{1,0}} \phi
$$

Proof. Let $\phi$ be a basic holomorphic form of type $(r, 0)$. Trivially, $\square_{B} \phi=0$. Hence from (3.38), the proof follows.

Corollary 3.20. (First proved in [10, Théorème 3.4.6]) Let $\left(M, g_{Q}, \mathcal{F}, J\right)$ be a minimal transversely Kähler foliation with compact leaf closures on a Riemannian manifold with bundlelike metric $g_{M}$. Then a basic form of type $(r, 0)$ is basic-harmonic if and only if it is basic holomorphic.

Proof. Let $\phi$ be a basic form of type $(r, 0)$. Since automatically $\bar{\partial}_{B}^{*} \phi=0, \bar{\partial}_{B} \phi=0$ if and only if $\bar{\square}_{B} \phi=0$, if and only if $\Delta_{B} \phi=0$ by Proposition 3.19 ,

Note that $\bar{\partial}_{B}^{2}=0$. So there exists a complex

$$
0 \stackrel{\bar{\partial}_{B}}{\longrightarrow} \cdots \stackrel{\bar{\partial}_{B}}{\longrightarrow} \Omega_{B}^{r, s-1}(\mathcal{F}) \stackrel{\bar{\partial}_{B}}{\longrightarrow} \Omega_{B}^{r, s}(\mathcal{F}) \stackrel{\bar{\partial}_{B}}{\longrightarrow} \Omega_{B}^{r, s+1}(\mathcal{F}) \stackrel{\bar{\partial}_{B}}{\longrightarrow} \cdots \stackrel{\bar{\partial}_{B}}{\longrightarrow} 0 .
$$

Hence we can define the basic Dolbeault cohomology group on a transversely holomorphic foliation by

$$
H_{B}^{r, s}=\frac{\operatorname{ker} \bar{\partial}_{B}}{\operatorname{Im} \bar{\partial}_{B}}
$$

Then we have the generalization of the Dolbeault decomposition.

Theorem 3.21. (Proved in [10, Théorème 3.3.3]) Let $\left(M, g_{Q}, \mathcal{F}, J\right)$ be a transversely Hermitian foliation on a closed Riemannian manifold with bundle-like metric $g_{M}$. Then

$$
\Omega_{B}^{r, s}(\mathcal{F}) \cong \mathcal{H}_{B}^{r, s} \oplus \operatorname{Im} \bar{\partial}_{B} \oplus \operatorname{Im} \bar{\partial}_{B}^{*},
$$

where $\mathcal{H}_{B}^{r, s}=\operatorname{ker} \bar{\square}_{B}$ is finite dimensional. Moreover, $\mathcal{H}_{B}^{r, s} \cong H_{B}^{r, s}$. 
Proof. The proof is similar to the one in Theorem [2.2, See [24] precisely.

Let $\square_{T}=\partial_{T} \partial_{T}^{*}+\partial_{T}^{*} \partial_{T}$ and $\bar{\square}_{T}=\bar{\partial}_{T} \bar{\partial}_{T}^{*}+\bar{\partial}_{T}^{*} \bar{\partial}_{T}$. Then from (3.16) and (3.17), which only require the transverse Hermitian structure,

$$
\bar{*} \bar{\square}_{B}=\square_{T} \bar{*}, \quad \bar{*} \square_{B}=\bar{\square}_{T} \bar{*} .
$$

Theorem 3.22. Let $\left(M, g_{Q}, \mathcal{F}, J\right)$ be a transversely Hermitian foliation on a closed Riemannian manifold with bundle-like metric $g_{M}$. Then

$$
\mathcal{H}_{B}^{r, s} \cong \mathcal{H}_{T}^{n-r, n-s}
$$

where $\mathcal{H}_{T}^{r, s}=\operatorname{ker} \bar{\square}_{T}$.

Proof. We define the operator $\sharp: \Omega_{B}^{r, s}(\mathcal{F}) \rightarrow \Omega_{B}^{n-r, n-s}(\mathcal{F})$ by

$$
\sharp \phi:=\bar{*} \bar{\phi} \text {. }
$$

From (3.54), we have $\sharp \bar{\square}_{B} \phi=\bar{\square}_{T}(\sharp \phi)$. Hence if $\bar{\square}_{B} \phi=0$, then $\bar{\square}_{T}(\sharp \phi)=0$. This means that $\sharp \phi \in \operatorname{ker} \bar{\square}_{T}$. Since $\bar{*}$ is an isomorphism, it is clear that $\sharp$ is also an isomorphism. Hence (3.55) is proved.

Let $H_{T}^{r, s}$ denote the basic cohomology corresponding to the differential $\partial_{T}$ restricted to basic forms of type $(r, s)$. This is a type of basic Lichnerowicz cohomology. The interested reader may consult [3, Section 3] and [34, Section 3, called "adapted cohomology" here] for information about ordinary Lichnerowicz cohomology and [1] for the basic case. The following corollary follows directly from the appropriate analogues of the Hodge theorems Theorem 2.2 and Theorem 3.21, A similar theorem was proved in 23] for ordinary basic cohomology.

Corollary 3.23. Let $\left(M, g_{Q}, \mathcal{F}, J\right)$ be a transversely Hermitian foliation on a closed Riemannian manifold with bundle-like metric $g_{M}$. Then

$$
H_{B}^{r, s} \cong H_{T}^{n-r, n-s}
$$

If $\mathcal{F}$ is minimal, then $\bar{\square}_{B}=\bar{\square}_{T}$. Hence we have the following corollary.

Corollary 3.24. (See reference below.) If $\left(M, g_{Q}, \mathcal{F}, J\right)$ is a minimal transversely Hermitian foliation on a closed Riemannian manifold with bundle-like metric $g_{M}$, then Kodaira-Serre duality holds:

$$
\mathcal{H}_{B}^{r, s} \cong \mathcal{H}_{B}^{n-r, n-s}
$$

Remark 3.25. The Theorem above was first proved in [10, Theorem 3.3.4]. In Theorem 3.4.6 of the same paper, it was also shown that if in addition the foliation is minimal and Kähler, $\mathcal{H}_{B}^{r, s} \cong \mathcal{H}_{B}^{s, r}, \mathcal{H}_{B}^{k}=\bigoplus_{r+s=k} \mathcal{H}_{B}^{r, s}$ and that $\mathcal{H}_{B}^{r, r} \neq\{0\}$ for all $r$.

Example 3.26. In general, Kodaira-Serre duality (3.56) fails for a transversely Kähler foliation on a closed Riemannian manifold with bundle-like metric, even though the standard 
version holds on any compact complex manifold. We return to the transversely Kähler foliation of Example 3.1, with the same notation. One can easily verify that

$$
\begin{aligned}
& H_{B}^{0,0}=\operatorname{ker} \bar{\partial}_{B}^{0,0}=\operatorname{span}\{1\} \\
& H_{B}^{1,0}=\operatorname{ker} \bar{\partial}_{B}^{1,0}=\{0\} \\
& H_{B}^{0,1}=\frac{\operatorname{ker} \bar{\partial}_{B}^{0,1}}{\operatorname{im} \bar{\partial}_{B}^{0,0}}=\operatorname{span}\left\{Y_{1}^{*}-i Y_{2}^{*}\right\} \\
& H_{B}^{1,1}=\frac{\Omega_{B}^{1,1}}{\operatorname{im} \bar{\partial}_{B}^{1,0}}=\{0\},
\end{aligned}
$$

where the last equality is true because one can show that every element of $\Omega_{B}^{1,1}$ is $\bar{\partial}_{B}$-exact. Then observe that the ordinary basic cohomology Betti numbers for this foliation are $h_{B}^{0}=$ $h_{B}^{1}=1, h_{B}^{2}=0$, we see that the basic Dolbeault Betti numbers satisfy

$$
h_{B}^{0,0}=h_{B}^{0,1}=1, \quad h_{B}^{1,0}=h_{B}^{1,1}=0 .
$$

So even though it is true that

$$
h_{B}^{j}=\sum_{p+q=j} h_{B}^{p, q}
$$

and the foliation is transversely Kähler, we also have (with $n=1$ )

$$
h_{B}^{p, q} \neq h_{B}^{q, p}, \quad h_{B}^{r, s} \neq h_{B}^{n-r, n-s} .
$$

The exactness of the basic Kähler form causes the Kodaira-Serre argument, the Lefschetz theorem, the Hodge diamond ideas to fail. Thus, for a nontaut, transversely Kähler foliation, it is not necessarily true that the odd basic Betti numbers are even, and the basic Dolbeault numbers do not have the same kinds of symmetries as Dolbeault cohomology on Kähler manifolds. Also, this example shows that the even degree basic cohomology groups are not always nonzero, as is the case for ordinary cohomology for symplectic manifolds (and thus all Kähler manifolds). However, these analogous results really are true in the minimal case, and in fact there is an analogue of the Lefschetz decomposition for basic cohomology (see [10, Section 3.4.7]).

\section{The WeitzenböcK Formula}

Let $\left(M, g_{Q}, \mathcal{F}, J\right)$ be a transversely Kähler foliation with compact leaf closures on a Riemannian manifold with bundle-like metric $g_{M}$. We define two operators

$$
\begin{aligned}
& \nabla_{T}^{*} \nabla_{T} \phi=-\sum_{a} \nabla_{V_{a}} \nabla_{\bar{V}_{a}} \phi+\nabla_{H^{0,1}} \phi, \\
& \bar{\nabla}_{T}^{*} \bar{\nabla}_{T} \phi=-\sum_{a} \nabla_{\bar{V}_{a}} \nabla_{V_{a}} \phi+\nabla_{H^{1,0}} \phi .
\end{aligned}
$$

Then by direct calculation, we have

$$
\nabla_{T}^{*} \nabla_{T} \phi=\bar{\nabla}_{T}^{*} \bar{\nabla}_{T} \phi+\nabla_{H^{0,1}-H^{1,0}} \phi+\sum_{a} R^{Q}\left(\bar{V}_{a}, V_{a}\right) \phi
$$

for any basic form $\phi$. And we have the following proposition. 
Proposition 4.1. [19, Proposition 3.2] Let $\left(M, g_{Q}, \mathcal{F}, J\right)$ be a transversely Kähler foliation on a compact Riemannian manifold with bundle-like metric $g_{M}$. Then the operators $\nabla_{T}^{*} \nabla_{T}$ and $\bar{\nabla}_{T}^{*} \bar{\nabla}_{T}$ are formally self-adjoint and positive-definite.

Proof. Fix $x \in M$ and choose a normal frame field $\left\{V_{a}\right\}$ of type $(1,0)$ such that $\left(\nabla V_{a}\right)_{x}=$ 0 . Then for any $\phi, \psi \in \Omega_{B}^{r, s}(\mathcal{F})$,

$$
\begin{aligned}
\ll \nabla_{T}^{*} \nabla_{T} \phi, \psi \gg= & \int_{M}\left\langle-\sum_{a} \nabla_{V_{a}} \nabla_{\bar{V}_{a}} \phi+\nabla_{H^{0,1}} \phi, \psi\right\rangle \\
= & -\int_{M} \sum_{a} V_{a}\left\langle\nabla_{\bar{V}_{a}} \phi, \psi\right\rangle+\int_{M} \sum_{a}\left\langle\nabla_{\bar{V}_{a}} \phi, \nabla_{\bar{V}_{a}} \psi\right\rangle+\int_{M}\left\langle\nabla_{H^{0,1}} \phi, \psi\right\rangle \\
= & -\int_{M}\left\langle\phi, \sum_{a} \nabla_{V_{a}} \nabla_{\bar{V}_{a}} \psi\right\rangle+\int_{M}\left\langle\nabla_{H^{0,1}} \phi, \psi\right\rangle \\
& -\int_{M} \sum_{a} V_{a}\left\langle\nabla_{\bar{V}_{a}} \phi, \psi\right\rangle+\int_{M} \sum_{a} \bar{V}_{a}\left\langle\phi, \nabla_{\bar{V}_{a}} \psi\right\rangle .
\end{aligned}
$$

If we take complex vector fields $Y, Z \in Q^{C}$ satisfying

$$
g_{Q}(Y, X)=\left\langle\nabla_{X+i J X} \phi, \psi\right\rangle, \quad g_{Q}(Z, X)=\left\langle\phi, \nabla_{X+i J X} \psi\right\rangle
$$

for any vector field $X \in \Gamma Q$, then by the transversal divergence theorem (Theorem 2.8),

$$
\begin{aligned}
\int_{M} \sum_{a} V_{a}\left\langle\nabla_{\bar{V}_{a}} \phi, \psi\right\rangle & =\frac{1}{2} \int_{M} \operatorname{div}_{\nabla} Y=\int_{M}\left\langle\nabla_{H^{0,1}} \phi, \psi\right\rangle, \\
\int_{M} \sum_{a} \bar{V}_{a}\left\langle\phi, \nabla_{\bar{V}_{a}} \psi\right\rangle & =\frac{1}{2} \int_{M} \operatorname{div}_{\nabla} Z=\int_{M}\left\langle\phi, \nabla_{H^{0,1}} \psi\right\rangle .
\end{aligned}
$$

From the equations above,

$$
\begin{aligned}
\ll \nabla_{T}^{*} \nabla_{T} \phi, \psi \gg & =\int_{M}\left\langle\nabla_{\mathrm{T}} \phi, \nabla_{\mathrm{T}} \psi\right\rangle \\
& =\int_{M}\left\langle\phi,-\sum_{a} \nabla_{V_{a}} \nabla_{\bar{V}_{a}} \psi\right\rangle+\int_{M}\left\langle\phi, \nabla_{H^{0,1}} \psi\right\rangle \\
& =\ll \phi, \nabla_{T}^{*} \nabla_{T} \psi \gg,
\end{aligned}
$$

where $\left\langle\nabla_{\mathrm{T}} \phi, \nabla_{\mathrm{T}} \psi\right\rangle=\sum_{a}\left\langle\nabla_{\bar{V}_{a}} \phi, \nabla_{\bar{V}_{a}} \psi\right\rangle$. Hence the proof is complete. Others are similarly proved.

Proposition 4.2. If $\left(M, g_{Q}, \mathcal{F}, J\right)$ is a transversely Kähler foliation with compact leaf closures on a Riemannian manifold with bundle-like metric $g_{M}$, we have for all $\phi \in \Omega_{B}^{r, s}(\mathcal{F})$,

$$
\begin{aligned}
& \left.\left.\bar{\square}_{B} \phi=\nabla_{T}^{*} \nabla_{T} \phi+\sum_{a, b} \bar{\omega}^{a} \wedge \bar{V}_{b}\right\lrcorner R^{Q}\left(V_{b}, \bar{V}_{a}\right) \phi+\sum_{a} \bar{\omega}^{a} \wedge\left(\nabla_{\bar{V}_{a}} H^{0,1}\right)\right\lrcorner \phi, \\
& \left.\left.\square_{B} \phi=\bar{\nabla}_{T}^{*} \bar{\nabla}_{T} \phi+\sum_{a, b} \omega^{a} \wedge V_{b}\right\lrcorner R^{Q}\left(\bar{V}_{b}, V_{a}\right) \phi+\sum_{a} \omega^{a} \wedge\left(\nabla_{V_{a}} H^{1,0}\right)\right\lrcorner \phi .
\end{aligned}
$$

Proof. Fix $x \in M$. Observe that since $(M, \mathcal{F})$ is transversely Kähler, a local $J$-basic frame $\left\{E_{a}, J E_{a}\right\}_{a=1, \ldots, n}$ can be chosen to consist of basic fields, so that the corresponding $V_{a}$ and 
$\omega_{a}$ are also basic fields of type $(1,0)$. Furthermore, at $x \in M$, we may make the choices so that $\left(\nabla V_{a}\right)_{x}=0$. Then at $x$, by (3.10) and Proposition 3.6 we have for all $\phi \in \Omega_{B}^{r, s}(\mathcal{F})$,

$$
\begin{aligned}
\bar{\partial}_{B} \bar{\partial}_{B}^{*} \phi= & \left.\left.-\sum_{a, b} \bar{\omega}^{a} \wedge \bar{V}_{b}\right\lrcorner \nabla_{\bar{V}_{a}} \nabla_{V_{b}} \phi+\sum_{a} \bar{\omega}^{a} \wedge\left(\nabla_{\bar{V}_{a}} H^{0,1}\right)\right\lrcorner \phi \\
& \left.+\sum_{a} \bar{\omega}^{a} \wedge H^{0,1}\right\lrcorner \nabla_{\bar{V}_{a}} \phi .
\end{aligned}
$$

Similarly, we have

$$
\begin{aligned}
\bar{\partial}_{B}^{*} \bar{\partial}_{B} \phi= & \left.-\sum_{a} \nabla_{V_{a}} \nabla_{\bar{V}_{a}} \phi+\nabla_{H^{0,1}} \phi+\sum_{a, b} \bar{\omega}^{a} \wedge \bar{V}_{b}\right\lrcorner \nabla_{V_{b}} \nabla_{\bar{V}_{a}} \phi \\
& \left.-\sum_{b} \bar{\omega}^{a} \wedge H^{0,1}\right\lrcorner \nabla_{\bar{V}_{a}} \phi .
\end{aligned}
$$

By summing the two equation above, we have (4.6). The proof of (4.7) is similarly proved.

The analogous results of the proposition above for the case of the twisted basic Dolbeault Laplacians was shown in [14, Theorem 3.1].

Proposition 4.3. If $\left(M, g_{Q}, \mathcal{F}, J\right)$ is a transversely Kähler foliation with compact leaf closures on a Riemannian manifold with bundle-like metric $g_{M}$, the following hold:

(1) If $\phi$ is a basic form of type $(r, 0)$, then

$$
\bar{\square}_{B} \phi=\nabla_{T}^{*} \nabla_{T} \phi .
$$

(2) If $\phi$ is a basic form of type $(0, s)$, then

$$
\square_{B} \phi=\bar{\nabla}_{T}^{*} \bar{\nabla}_{T} \phi
$$

(3) If $\phi$ is a basic form of type $(r, n)$, then

$$
\begin{aligned}
\bar{\square}_{B} \phi & =\nabla_{T}^{*} \nabla_{T} \phi+\sum_{a} R^{Q}\left(V_{a}, \bar{V}_{a}\right) \phi+\operatorname{div}_{\nabla}\left(H^{0,1}\right) \phi \\
& =\bar{\nabla}_{T}^{*} \bar{\nabla}_{T} \phi-i \nabla_{J \kappa_{B}^{\sharp}} \phi+\operatorname{div}_{\nabla}\left(H^{0,1}\right) \phi .
\end{aligned}
$$

(4) If $\phi$ is a basic form of type $(n, s)$, then

$$
\square_{B} \phi=\nabla_{T}^{*} \nabla_{T} \phi+i \nabla_{\kappa_{B}^{\sharp}} \phi+\operatorname{div}_{\nabla}\left(H^{1,0}\right) \phi .
$$

Proof. (1) Let $\phi$ be a basic form of type $(r, 0)$. Then $\left.\bar{V}_{b}\right\lrcorner R^{Q}\left(V_{b}, \bar{V}_{a}\right) \phi$ and $\left.\left(\nabla_{\bar{V}_{a}} H^{0,1}\right)\right\lrcorner \phi$ are of type $(r,-1)$, which are zero. Hence from (4.6), Eq.(4.8) is proved.

(2) The proof of (4.9) follows from the conjugation of (4.8).

(3) Now, let $\phi$ be of type $(r, n)$. Then $\left.\bar{\omega}^{a} \wedge \bar{V}_{b}\right\lrcorner \phi=\delta_{b}^{a} \phi$, so that $\operatorname{div}_{\nabla}\left(H^{0,1}\right) \phi=\sum_{a} \bar{\omega}^{a} \wedge$ $\left.\left(\nabla_{\bar{V}_{a}} H^{0,1}\right)\right\lrcorner \phi$. Since $R^{Q}\left(V_{b}, \bar{V}_{a}\right) \phi$ is also of type $(r, n)$, we have

$$
\left.\sum_{a, b} \omega^{a} \wedge V_{b}\right\lrcorner R^{Q}\left(V_{b}, \bar{V}_{a}\right) \phi=\sum_{a} R^{Q}\left(V_{a}, \bar{V}_{a}\right) \phi .
$$

From (4.6) and (4.13), Eq. (4.10) is proved. Statement (4.11) follows directly from (4.3).

(4) The proof of (4.12) follows from the conjugation of (4.11). 
Proposition 4.4. Let $\left(M, g_{Q}, \mathcal{F}, J\right)$ be a transversely Kähler foliation with compact leaf closures on a Riemannian manifold with bundle-like metric $g_{M}$. Then for all $\phi \in \Omega_{B}^{r, s}(\mathcal{F})$,

$$
\begin{aligned}
\frac{1}{2} \Delta_{B}|\phi|^{2}= & \left\langle\nabla_{T}^{*} \nabla_{T} \phi, \phi\right\rangle+\left\langle\phi, \bar{\nabla}_{T}^{*} \bar{\nabla}_{T} \phi\right\rangle-\sum_{a=1}^{n}\left\{\left|\nabla_{\bar{V}_{a}} \phi\right|^{2}+\left|\nabla_{V_{a}} \phi\right|^{2}\right\} \\
& +\frac{1}{2}\left(H^{1,0}-H^{0,1}\right)|\phi|^{2},
\end{aligned}
$$

where $|\phi|^{2}=\langle\phi, \phi\rangle$.

Proof. Let $\phi \in \Omega_{B}^{r, s}(\mathcal{F})$. Since the connection $\nabla$ is Hermitian, from (4.8)

$$
\begin{aligned}
\bar{\square}_{B}|\phi|^{2} & =-\sum_{a} \nabla_{V_{a}} \nabla_{\bar{V}_{a}}|\phi|^{2}+\nabla_{H^{0,1}}|\phi|^{2} \\
& =\left\langle\nabla_{T}^{*} \nabla_{T} \phi, \phi\right\rangle+\left\langle\phi, \bar{\nabla}_{T}^{*} \bar{\nabla}_{T} \phi\right\rangle-\sum_{a=1}^{n}\left\{\left|\nabla_{\bar{V}_{a}} \phi\right|^{2}+\left|\nabla_{V_{a}} \phi\right|^{2}\right\} .
\end{aligned}
$$

From Corollary 3.13 (3.43) and (3.44), we have

$$
\Delta_{B}|\phi|^{2}=2 \bar{\square}_{B}|\phi|^{2}-i J \kappa_{B}^{\sharp}\left(|\phi|^{2}\right) .
$$

Since $i J \kappa_{B}^{\sharp}=H^{0,1}-H^{1,0}$, the proof follows.

From Proposition 4.4, we have the following corollary.

Corollary 4.5. If $\phi$ is a basic form of type $(r, 0)$, then

$$
\begin{aligned}
-\frac{1}{2} \Delta_{B}|\phi|^{2}= & -\left\langle\bar{\square}_{B} \phi, \phi\right\rangle-\left\langle\phi, \bar{\square}_{B} \phi\right\rangle+\sum_{a}\left\{\left|\nabla_{\bar{V}_{a}} \phi\right|^{2}+\left|\nabla_{V_{a}} \phi\right|^{2}\right\} \\
& +\sum_{a}\left\langle\phi, R^{Q}\left(\bar{V}_{a}, V_{a}\right) \phi\right\rangle-\frac{1}{2}\left\langle\nabla_{H^{1,0}-H^{0,1}} \phi, \phi\right\rangle-\frac{1}{2}\left\langle\phi, \nabla_{H^{1,0}-H^{0,1}} \phi\right\rangle .
\end{aligned}
$$

Proof. The proof follows from (4.3) and (4.8).

Corollary 4.6. If $\phi$ is a basic-harmonic form of type $(r, 0)$, then

$$
\begin{aligned}
-\frac{1}{2} \Delta_{B}|\phi|^{2}= & \sum_{a}\left\{\left|\nabla_{\bar{V}_{a}} \phi\right|^{2}+\left|\nabla_{V_{a}} \phi\right|^{2}\right\}+\left\langle\phi, \sum_{a} R^{Q}\left(\bar{V}_{a}, V_{a}\right) \phi\right\rangle \\
& \left.\left.+\frac{1}{2} \sum_{a}\left\{\left\langle\omega^{a} \wedge\left(\nabla_{V_{a}} H^{1,0}\right)\right\lrcorner \phi, \phi\right\rangle+\left\langle\phi, \omega^{a} \wedge\left(\nabla_{V_{a}} H^{1,0}\right)\right\lrcorner \phi\right\rangle\right\} .
\end{aligned}
$$

Proof. Let $\phi$ be a basic-harmonic form of type $(r, 0)$. From (3.38), we have

$$
\begin{aligned}
& \left.\left.\left.2 \bar{\square}_{B} \phi=-\partial_{B} H^{1,0}\right\lrcorner \phi-H^{1,0}\right\lrcorner \partial_{B} \phi+H^{0,1}\right\lrcorner \bar{\partial}_{B} \phi, \\
& \left.\left.\bar{\partial}_{B} H^{1,0}\right\lrcorner \phi+H^{1,0}\right\lrcorner \bar{\partial}_{B} \phi=0 .
\end{aligned}
$$


Hence from Corollary 4.5, we have

$$
\begin{aligned}
-\frac{1}{2} \Delta_{B}|\phi|^{2} & \left.\left.\left.=\frac{1}{2}\left\langle\partial_{B} H^{1,0}\right\lrcorner \phi+H^{1,0}\right\lrcorner \partial_{B} \phi-H^{0,1}\right\lrcorner \bar{\partial}_{B} \phi, \phi\right\rangle \\
& \left.\left.\left.+\frac{1}{2}\left\langle\phi, \partial_{B} H^{1,0}\right\lrcorner \phi+H^{1,0}\right\lrcorner \partial_{B} \phi-H^{0,1}\right\lrcorner \bar{\partial}_{B} \phi\right\rangle \\
& -\frac{1}{2}\left\langle\nabla_{H^{1,0}-H^{0,1}} \phi, \phi\right\rangle-\frac{1}{2}\left\langle\phi, \nabla_{H^{1,0}-H^{0,1}} \phi\right\rangle \\
& +\sum_{a}\left\{\left|\nabla_{\bar{V}_{a}} \phi\right|^{2}+\left|\nabla_{V_{a}} \phi\right|^{2}\right\}+\left\langle\phi, \sum_{a} R^{Q}\left(\bar{V}_{a}, V_{a}\right) \phi\right\rangle .
\end{aligned}
$$

On the other hand, since $\phi$ is of type $(r, 0)$, we have

$$
\begin{aligned}
& \left.H^{0,1}\right\lrcorner \bar{\partial}_{B} \phi=\nabla_{H^{0,1}} \phi, \\
& \left.\left.\left.\partial_{B} H^{1,0}\right\lrcorner \phi+H^{1,0}\right\lrcorner \partial_{B} \phi=\nabla_{H^{1,0}} \phi+\sum_{a} \omega^{a} \wedge\left(\nabla_{V_{a}} H^{1,0}\right)\right\lrcorner \phi .
\end{aligned}
$$

Hence from these two equations, we have

$$
\begin{aligned}
-\frac{1}{2} \Delta_{B}|\phi|^{2}= & \sum_{a}\left\{\left|\nabla_{\bar{V}_{a}} \phi\right|^{2}+\left|\nabla_{V_{a}} \phi\right|^{2}\right\}+\left\langle\phi, \sum_{a} R^{Q}\left(\bar{V}_{a}, V_{a}\right) \phi\right\rangle \\
& \left.\left.+\frac{1}{2} \sum_{a}\left\{\left\langle\omega^{a} \wedge\left(\nabla_{V_{a}} H^{1,0}\right)\right\lrcorner \phi, \phi\right\rangle+\left\langle\phi, \omega^{a} \wedge\left(\nabla_{V_{a}} H^{1,0}\right)\right\lrcorner \phi\right\rangle\right\} .
\end{aligned}
$$

Hence the proof is complete.

Remark 4.7. Let $\xi$ and $\eta$ be basic forms of type $(1,0)$, i.e., $\xi, \eta \in \Omega_{B}^{1,0}(\mathcal{F})$. Then

$$
\left\langle R^{Q}\left(\bar{V}_{a}, V_{a}\right) \xi, \eta\right\rangle=\left\langle\xi, R^{Q}\left(\bar{V}_{a}, V_{a}\right) \eta\right\rangle
$$

That is, $\sum_{a} R^{Q}\left(\bar{V}_{a}, V_{a}\right)$ is Hermitian symmetric, and so it is diagonalized by $\left\{\omega^{a}\right\}$. Let $\lambda_{a}$ be a real eigenvalue of $\sum_{a} R^{Q}\left(\bar{V}_{a}, V_{a}\right)$ corresponding to $\omega^{a}$, i.e., $\left(\sum_{a} R^{Q}\left(\bar{V}_{a}, V_{a}\right)\right) \omega^{b}=\lambda_{b} \omega^{b}$. Then by using the first Bianchi identity, we have

$$
\begin{aligned}
\lambda_{a} & =\left\langle\sum_{b} R^{Q}\left(\bar{V}_{b}, V_{b}\right) \omega^{a}, \omega^{a}\right\rangle \\
& =\sum_{b=1}^{n} g_{Q}\left(R^{Q}\left(\bar{V}_{b}, V_{b}\right) V_{a}, \bar{V}_{a}\right) \\
& =\operatorname{Ric}^{Q}\left(E_{a}, E_{a}\right) .
\end{aligned}
$$

From Remark 4.7, we have the following corollary.

Corollary 4.8. Let $\phi$ be a basic-harmonic form of type $(r, 0)$. Then

$$
\begin{aligned}
-\frac{1}{2} \Delta_{B}|\phi|^{2}= & \sum_{a}\left\{\left|\nabla_{\bar{V}_{a}} \phi\right|^{2}+\left|\nabla_{V_{a}} \phi\right|^{2}\right\}+\sum_{i=1}^{r} \operatorname{Ric}^{Q}\left(E_{a_{i}}, E_{a_{i}}\right)|\phi|^{2} \\
& \left.\left.+\frac{1}{2} \sum_{a}\left\{\left\langle\omega^{a} \wedge\left(\nabla_{V_{a}} H^{1,0}\right)\right\lrcorner \phi, \phi\right\rangle+\left\langle\phi, \omega^{a} \wedge\left(\nabla_{V_{a}} H^{1,0}\right)\right\lrcorner \phi\right\rangle\right\} .
\end{aligned}
$$


Proof. From Remark 4.7, we have that for any $\phi \in \Omega_{B}^{r, 0}(\mathcal{F})$,

$$
\left\langle\phi, \sum_{a} R^{Q}\left(\bar{V}_{a}, V_{a}\right) \phi\right\rangle=\left(\sum_{i=1}^{r} \lambda_{a_{i}}\right)|\phi|^{2}=\sum_{i=1}^{r} \operatorname{Ric}^{Q}\left(E_{a_{i}}, E_{a_{i}}\right)|\phi|^{2} .
$$

From Corollary 4.6, the proof is complete.

Theorem 4.9. Let $\mathcal{F}$ be a transversely Kähler foliation on a closed manifold with a bundlelike metric. If the transversal Ricci curvature is nonnegative and positive at some point, then for $r>0$ there are no non-zero basic-harmonic forms of type $(r, 0)$; i.e., $\mathcal{H}_{B}^{r, 0}=\{0\}$.

Proof. First, we have that for any basic form $\phi$,

$$
\begin{aligned}
\left.\sum_{a=1}^{n}\left\langle\omega^{a} \wedge\left(\nabla_{V_{a}} H^{1,0}\right)\right\lrcorner \phi, \phi\right\rangle & \left.\left.=\sum_{a, b=1}^{n} g_{Q}\left(\nabla_{V_{a}} H^{1,0}, \bar{V}_{b}\right)\left\langle V_{b}\right\lrcorner \phi, V_{a}\right\lrcorner \phi\right\rangle \\
& \left.=\sum_{a=1}^{n} g_{Q}\left(\nabla_{V_{a}} H^{1,0}, \bar{V}_{a}\right) \mid V_{a}\right\lrcorner\left.\phi\right|^{2} .
\end{aligned}
$$

Similarly, we have

$$
\left.\left.\sum_{a=1}^{n}\left\langle\phi, \omega^{a} \wedge\left(\nabla_{V_{a}} H^{1,0}\right)\right\lrcorner \phi\right\rangle=\sum_{a=1}^{n} g_{Q}\left(\nabla_{\bar{V}_{a}} H^{0,1}, V_{a}\right) \mid V_{a}\right\lrcorner\left.\phi\right|^{2} .
$$

Now, we put $\left.C=\min _{a, x} \mid V_{a}\right\lrcorner\left.\phi\right|^{2} \geq 0$. Then from the equations above, we have

$$
\begin{aligned}
& \left.\left.\sum_{a=1}^{n}\left\{\left\langle\omega^{a} \wedge\left(\nabla_{V_{a}} H^{1,0}\right)\right\lrcorner \phi, \phi\right\rangle+\left\langle\phi, \omega^{a} \wedge\left(\nabla_{V_{a}} H^{1,0}\right)\right\lrcorner \phi\right\rangle\right\} \\
& \quad \geq C \sum_{a=1}^{n}\left\{g_{Q}\left(\nabla_{V_{a}} H^{1,0}, \bar{V}_{a}\right)+\overline{g_{Q}\left(\nabla_{V_{a}} H^{1,0}, \bar{V}_{a}\right)}\right\} \\
& \quad \geq C \operatorname{div}_{\nabla}\left(\kappa_{B}^{\sharp}\right) .
\end{aligned}
$$

In the last inequality, we used $\sum_{a=1}^{n} g_{Q}\left(\nabla_{V_{a}} H^{1,0}, \bar{V}_{a}\right)=\operatorname{div}_{\nabla}\left(H^{1,0}\right)$. Since $\delta_{B} \kappa_{B}=-\operatorname{div}_{\nabla}\left(\kappa_{B}^{\sharp}\right)+$ $\left|\kappa_{B}\right|^{2}$ and $\delta_{B} \kappa_{B}=0$, we have

$$
\left.\left.\sum_{a=1}^{n}\left\{\left\langle\omega^{a} \wedge\left(\nabla_{V_{a}} H^{1,0}\right)\right\lrcorner \phi, \phi\right\rangle+\left\langle\phi, \omega^{a} \wedge\left(\nabla_{V_{a}} H^{1,0}\right)\right\lrcorner \phi\right\rangle\right\} \geq C\left|\kappa_{B}\right|^{2} .
$$

Let $\phi$ be a basic-harmonic form of type $(r, 0)$. Since the transversal Ricci curvature is nonnegative, from Corollary 4.8 and the inequality above, we have

$$
\Delta_{B}|\phi|^{2} \leq 0
$$

Hence by the maximum principle, $\phi$ is parallel. Since $\operatorname{Ric}^{Q}>0$ at some point, $\phi$ is zero.

Corollary 4.10. Let $\mathcal{F}$ be as in Theorem 4.9. If the transversal Ricci curvature is nonnegative and positive at some point, then for $r>0$ there are no non-zero basic holomorphic forms of type $(r, 0)$.

Proof. From 2.5, if the transversal Ricci curvature is nonnegative and positive at some point, then $\mathcal{H}_{B}^{1}=\{0\}$. Hence for some basic function $f, \kappa=d f$, which implies $\Delta_{B} f=$ 0 . Hence by the maximum principle, $h$ is constant. That is, $\kappa=0$. It follows from Corollary 3.20 , 
A theorem similar to the corollary above, with a stronger hypotheses on the transverse Ricci curvature, was proved in [14, Theorem 3.4].

\section{REFERENCES}

[1] H. Ait Haddou, Foliations and Lichnerowicz basic cohomology, Int. Math. Forum 2 (2007), no. 49-52, 2437-2446.

[2] J. A. Álvarez-López, The basic component of the mean curvature of Riemannian foliations, Ann. Global Anal. Geom. 10 (1992), 179-194.

[3] A. Banyaga, Some properties of locally conformal symplectic structures, Comment. Math. Helv. $7 \mathbf{7}$ (2002), no. 2, 383-398.

[4] C. P. Boyer and K. Galicki, Sasakian geometry. Oxford Mathematical Monographs, Oxford University Press, Oxford, 2008.

[5] C. P. Boyer, K. Galicki, and M. Nakamaye, On the geometry of Sasakian-Einstein 5-manifolds, Math. Ann. 325 (2003), no. 3, 485-524.

[6] B. Cappelletti-Montano, A. De Nicola, and I. Yudin, Hard Lefschetz Theorem for Sasakian manifolds J. Diff. Geom. 101 (2015), 47-66.

[7] Y. Carrière, Flots riemanniens, Astérisque 116 (1984), 31-52.

[8] D. Chinea, M. de León, M., and J. C. Marrero, Topology of cosymplectic manifolds, J. Math. Pures Appl. (9) $\mathbf{7 2}$ (1993), no. 6, 567-591.

[9] L. A. Cordero and R. A. Wolak, Properties of the basic cohomology of transversally Kähler foliations, Rendiconti del Circolo Matematico di Palermo, Serie II, 40 (1991), 177-188.

[10] A. El Kacimi-Alaoui, Opérateurs transversalement elliptiques sur un feuilletage riemannien et applications, Compositio Math. 73 (1990), no. 1, 57-106.

[11] A. El Kacimi-Alaoui and G. Hector, Décomposition de Hodge basique pour un feuilletage riemannien, Ann. Inst. Fourier 36, 3(1986), 207-227.

[12] O. Goertsches, H. Nozawa, and D. Töben, Dirk, Rigidity and vanishing of basic Dolbeault cohomology of Sasakian manifolds, J. Symplectic Geom. 14 (2016), no. 1, 31-70.

[13] G. Habib and K. Richardson, Modified differentials and basic cohomology for Riemannian foliations, J. Geom. Anal. 23 (2013), no. 3, 1314-1342.

[14] G. Habib and L. Vezzoni, Some remarks on Calabi-Yau and hyper-Kähler foliations, Differential Geom. Appl. 41 (2015), 12-32.

[15] J. Hebda, Curvature and focal points in Riemannian foliations, Indiana Univ. Math. J. 35 (1986), no. $2,321-331$.

[16] S. D. Jung, The first eigenvalue of the transversal Dirac operator, J. Geom. Phys. 39 (2001), 253-264.

[17] S. D. Jung and M. J. Jung, Transversally holomorphic maps between Kähler foliations, J. Math. Anal. Appl. 416 (2014), 683-697.

[18] S. D. Jung and H. Liu, Transversal infinitesimal automorphisms on Kähler foliations, Bull. Aust. Math. Soc. 86 (2012), 405-415.

[19] S. D. Jung and J. S. Pak, Some vanishing theorems on Kähler foliation, Comm. Korean Math. Soc. 11 (1996), 767-781.

[20] S. D. Jung and J. S. Pak, Some vanishing theorems on complete Kähler foliation Acta Math. Hungar. 77 (1997), no. 1-2, 15-28.

[21] S. D. Jung and K. Richardson, Transverse conformal Killing forms and a Gallot-Meyer theorem for foliations, Math. Z. 270 (2012), 337-350.

[22] F. W. Kamber and Ph. Tondeur, Duality for Riemannian foliations, Proc. Sympos. Pure Math., Amer. Math. Soc. 40 (1983), Part I, 609-618.

[23] F. W. Kamber and Ph. Tondeur, Duality theorems for foliations, Asterisque 116 (1984), 108-116.

[24] F. W. Kamber and Ph. Tondeur, De Rham-Hodge theory for Riemannian foliations, Math. Ann. 277 (1987), 415-431.

[25] Y. Kordyukov, M. Lejmi, and P. Weber, Seiberg-Witten invariants on manifolds with Riemannian foliations of codimension 4, J. Geom. Phys. 107 (2016), 114-135.

[26] P. March, M. Min-Oo and E. A. Ruh, Mean curvature of Riemannian foliations, Canad. Math. Bull. 39 (1996), 95-105 
[27] A. Mason, An application of stochastic flows to Riemannian foliations, Houston J. Math. 26 (2000), 481-515.

[28] M. Min-Oo, E. Ruh and Ph. Tondeur, Vanishing theorems for the basic cohomology of Riemannian foliations, J. Reine Angew. Math. 415 (1991), 167-174.

[29] A. Moroianu and L. Ornea, Eigenvalue estimates for the Dirac operator and harmonic 1-forms of constant length, C. R. Math. Acad. Sci. Paris 338 (2004), 561-564.

[30] S. Nisikawa and Ph. Tondeur, Transversal infinitesimal automorphisms for harmonic Kähler foliations, Tohoku Math. J. 40 (1988), 599-611.

[31] E. Park and K. Richardson, The basic Laplacian of a Riemannian foliation, Amer. J. Math. 118 (1996), 1249-1275.

[32] Ph. Tondeur, Foliations on Riemannian manifolds, Springer-Verlag, New-York, 1988.

[33] Ph. Tondeur, Geometry of foliations, Birkhäuser Verlag, 1997.

[34] I. Vaisman, Remarkable operators and commutation formulas on locally conformal Khler manifolds, Compositio Math. 40 (1980), no. 3, 287-299.

[35] R. Wolak, Sasakian structures: a foliated approach, arXiv:1605.04163 [math.DG].

[36] S. Yorozu and T. Tanemura, Green's theorem on a foliated Riemannian manifold and its applications, Acta Math. Hungarica 56 (1990), 239-245

Department of Mathematics, Jeju National University, Jeju 690-756, Republic of Korea E-mail address, S. D. Jung: sdjung@jejunu.ac.kr

Department of Mathematics, Texas Christian University, Fort Worth, Texas 76129, USA

E-mail address, K. Richardson: k.richardson@tcu.edu 